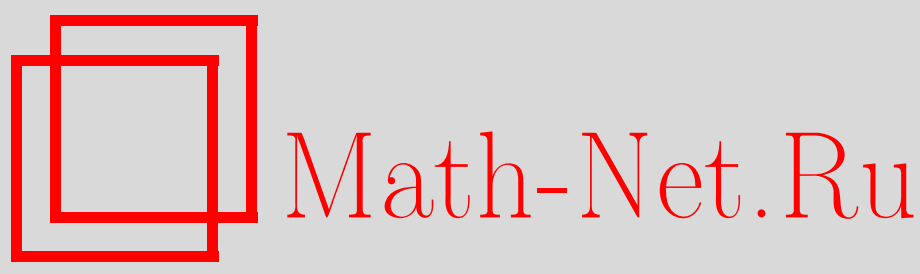

А. В. Пухликов, Бирационально жесткие многообразия с пучком двойных накрытий Фано. II, Матем. сб., 2004, том 195, номер 11, 119-156

DOI: https://doi.org/10.4213/sm861

Использование Общероссийского математического портала Math-Net.Ru подразумевает, что вы прочитали и согласны с пользовательским соглашением

http://www . mathnet.ru/rus/agreement

Параметры загрузки:

IP: 34.239 .49 .27

26 апреля 2023 г., 15:52:01 
УДК 513.6

\author{
А.В. Пухликов
}

\title{
Бирационально жесткие многообразия с пучком двойных накрытий Фано. II
}

\begin{abstract}
Продолжено изучение бирационалной геометрии расслоений $\Phi$ ано $\pi: V \rightarrow \mathbb{P}^{1}$, слой которых - двойная гиперповерхность Фано индекса 1. Для большинства семейств этого типа, не удовлетворяющих условию достаточной закрученности по базе, доказана бирациональная жесткость (в частности, отсутствие других структур расслоения на рационально связные многообразия) и вычислена группа бирациональных автоморфизмов. Существенно усилены основные компоненты метода максимальных особенностей, прежде всего, техника подсчета кратностей для расслоений $V / \mathbb{P}^{1}$ на многообразия Фано над прямой.

Библиограффия: 20 названий.
\end{abstract}

\section{Введение}

Настоящая статья является непосредственньм продолжением работы [1]. Мы изучаем бирациональную геометрию многомерных алгебраических многообразий с пучком двойных накрытий Фано, уже не предполагая выполненным стандартное условие достаточной закрученности по базе $-K^{2}$-условие [2], [3]. Как отмечено в [3], если отклонение от $K^{2}$-условия не слишком велико, техника метода максимальных особенностей сохраняет силу и позволяет доказьвать бирациональную жесткость. Этому и посвящена данная работа: рассматриваются расслоения $\Phi$ ано $V / \mathbb{P}^{1}$, не удовлетворяющие $K^{2}$-условию, однако отклоняющиеся от него не очень сильно.

Если отклонение от $K^{2}$-условия переходит некоторую границу, то многообразие $V$ перестает быть бирационально жестким. Нежесткие и "пограничные" семейства расслоений $V / \mathbb{P}^{1}$ будут рассмотрены в последующей статье - третьей части настоящего исследования. Для этого будет использована усиленная техника, развиваемая в настоящей работе.

0.1. $K^{2}$-условие и $K$-условие. В этой статье, как и в предыдущей работе [1], рассматриваются расслоения $\Phi$ ано $\pi: V \rightarrow \mathbb{P}^{1}$, удовлетворяющие условиям

$$
A^{1} V=\operatorname{Pic} V=\mathbb{Z} K_{V} \oplus \mathbb{Z} F, \quad A^{2} V=\mathbb{Z} K_{V}^{2} \oplus \mathbb{Z} H_{F}
$$

где $F$ - класс проекции $\pi, H_{F}=\left(-K_{V} \cdot F\right)$ - гиперплоское сечение слоя. Положим $A_{\mathbb{R}}^{1} V=A^{1} V \otimes \mathbb{R}, A_{\mathbb{R}}^{2} V=A^{2} V \otimes \mathbb{R}$. Пусть

$$
A_{+}^{1} V \subset A_{\mathbb{R}}^{1} V, \quad A_{\text {mov }}^{1} V \subset A_{\mathbb{R}}^{1} V, \quad A_{+}^{2} V \subset A_{\mathbb{R}}^{2} V
$$

Работа вьполнена в рамках Программы поддержки молодых российских ученых - докторов наук (грант № МД-328.2003.01) и при поддержке Фонда содействия отечественной науке.

(C) А. В. Пухликов 2004 
- замкнутые конусы, порожденные эффективными дивизорами, подвижными дивизорами и эффективньми циклами коразмерности два соответственно. Геометрия расслоения $V / \mathbb{P}^{1}$ сушественно зависит от взаимного расположения класса $K_{V}^{2}$ и конуса $A_{+}^{2} V$, а также взаимного расположения антиканонического класса $\left(-K_{V}\right)$ и конуса $A_{\mathrm{mov}}^{1} V$. Различные возможности для $K_{V}^{2}$ показаны на рис. $1-3$. Очевидно, $A_{\mathbb{R}}^{2} V=\mathbb{R} K_{V}^{2} \oplus \mathbb{R} H_{F}$, так что $A_{\mathbb{R}}^{2} V^{*}=\mathbb{R} \beta \oplus \mathbb{R} \chi$, где $\beta: A_{\mathbb{R}}^{2} V \rightarrow \mathbb{R}$ определяется условием $\beta\left(H_{F}\right)=1, \beta\left(K_{V}^{2}\right)=0$ и, аналогично, $\kappa\left(K_{V}^{2}\right)=1, \kappa\left(H_{F}\right)=0$.

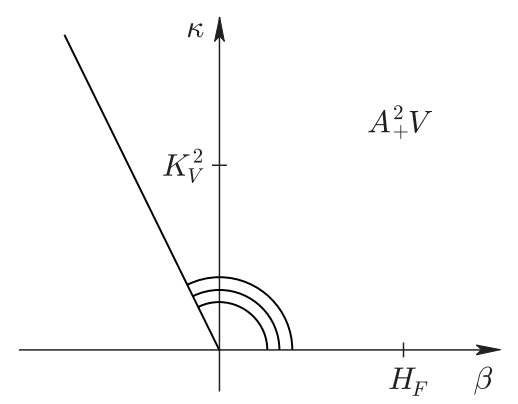

Рис. 1

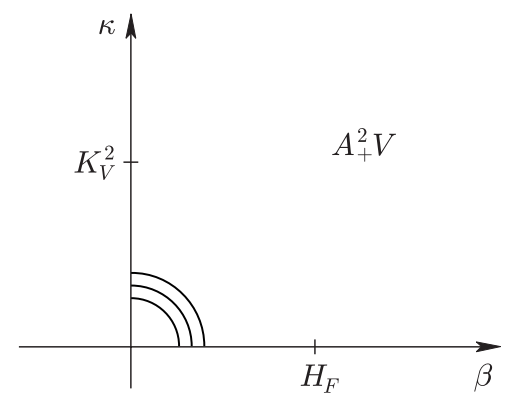

Рис. 2

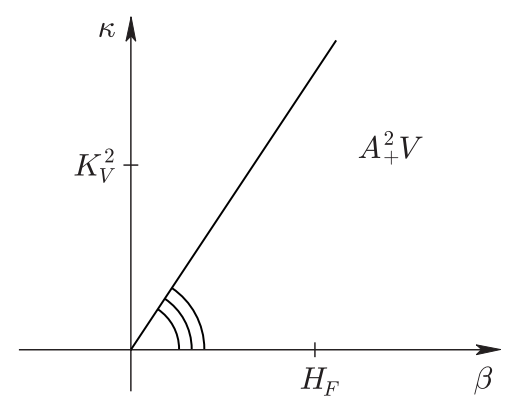

Рис. 3 
В случаях, изображенных на рис. 2,3 , выполнено $K^{2}$-условие:

$$
K_{V}^{2} \notin \operatorname{Int} A_{+}^{2} V \text {. }
$$

Хорошо известно (и легко доказать, см. [2], [3]), что из $K^{2}$-условия вытекает $K$-условие:

$$
-K_{V} \notin \operatorname{Int} A_{\mathrm{mov}}^{1} V .
$$

(Имеется естественное отображсение самопересечения

$$
\begin{gathered}
\mathrm{sq}: A_{\mathrm{mov}}^{1} V \rightarrow A_{+}^{2} V, \\
\mathrm{sq}: z \mapsto z^{2},
\end{gathered}
$$

причем легко проверить, что $\mathrm{sq}\left(\operatorname{Int} A_{\text {mov }}^{1} V\right) \subset \operatorname{Int} A_{+}^{2} V$, см. замечание 1.1 в $\S 1$ ниже.) Настоящая работа посвяшена расслоениям Фано $V / \mathbb{P}^{1}$, каждьй слой $F_{t}=\pi^{-1}(t)$ которых есть регулярная двойная гиперповерхность Фано индекса 1 , $F_{t} \in \mathscr{F}^{\mathrm{reg}}$, см. [1], причем $K^{2}$-условие не выполнено, т.е. $K_{V}^{2} \in \operatorname{Int} A_{+}^{2} V$ (рис. 1 ). Степень отклонения от $K^{2}$-условия измеряется числом $a \geqslant 0$, удовлетворяющим формуле

$$
K_{V}^{2}-a H_{F} \in \partial A_{+}^{2} V
$$

В настоящей статье рассматриваются в основном расслоения $V / \mathbb{P}^{1}$, удовлетворяющие $K$-условию. $K$-условие также не является необходимьм для бирациональной жесткости: если отклонение от $K$-условия не слишком велико, техника метода максимальных особенностей сохраняет силу и многообразие все еще поддается изучению. Семейства, не удовлетворяющие $K$-условию, а также некоторые другие, не рассматриваемые здесь и не рассмотренные в [1], будут изучены в последующей статье - третьей части работы.

0.2. Список рассматриваемых многообразий. Напомним конструкцию многообразий с пучком двойных гиперповерхностей Фано, более подробно см. [4], [1]. Пусть $\mathscr{E}=\bigoplus \mathscr{O}_{\mathbb{P}^{1}}\left(a_{i}\right)$ - локально свободный пучок ранга $M+2$, где $a_{0}=0 \leqslant a_{1} \leqslant \cdots \leqslant a_{M+1}, X=\mathbb{P}(\mathscr{E}), \operatorname{Pic} X=\mathbb{Z} L_{X} \oplus \mathbb{Z} R, \quad L_{X}$ - класс тавтологического пучка, $R$ - класс слоя естественной проекции $\pi_{X}: X \rightarrow \mathbb{P}^{1}$. Теперь многообразие $V$ реализуется как двойное накрытие $\sigma: V \rightarrow Q$ гладкой гиперповерхности $Q \subset X$,

$$
Q \sim m L_{X}+a_{Q} R, \quad a_{Q} \in \mathbb{Z}_{+},
$$

разветвленное над гладким дивизором $W_{Q}=W \cap Q$, где $W \subset X$,

$$
W \sim 2 l L_{X}+2 a_{W} R, \quad a_{W} \in \mathbb{Z}_{+} .
$$

Символом $\pi: V \rightarrow \mathbb{P}^{1}$ обозначаем естественную проекцию, символом $F_{t}-$ слой $\pi^{-1}(t)$, иногда опуская $t$. Легко видеть, что

$$
K_{V}=-L_{V}+\left(a_{X}+a_{Q}+a_{W}-2\right) F,
$$

где $L_{V}=\sigma^{*}\left(\left.L_{X}\right|_{Q}\right), a_{X}=a_{1}+\cdots+a_{M+1}$. Поскольку линейная система $\left|L_{X}\right|$ (и, значит, $\left|L_{V}\right|$ ) свободна, $K^{2}$-условие является следствием неравенства

$$
\left(K_{V}^{2} \cdot L_{V}^{M-1}\right)=2 m\left(4-a_{X}-2 a_{Q}-2 a_{W}\right)+2 a_{Q} \leqslant 0 .
$$


В настоящей работе рассматриваются семейства, не удовлетворяющие (1). Приведем их список. Параметры семейств записьваем в следуюшем формате:

$$
\left(\left(a_{1}, \ldots, a_{M+1}\right),\left(a_{Q}, a_{W}\right)\right),
$$

для компактности записи в наборе $\left(a_{1}, \ldots, a_{M+1}\right)$ пишем только ненулевые числа, если таковые имеются, в противном случае пишем (0): таким образом, например, (1) обозначает набор $(0, \ldots, 0,1),(1,1)$ обозначает набор $(0, \ldots, 0,1,1)$, а (0) обозначает набор $(0, \ldots, 0)$, целиком состоящий из нулей. Вот список многообразий, изученных в работе:
1. $((0),(2,0))$;
2. $((0),(1,1))$;
3. $((1),(0,1))$;
4. $((2),(1,0))$;
5. $((2),(0,0))$;
6. $((3),(0,0))$;
7. $((1,2),(0,0))$
8. $((1,1,1),(0,0))$.

Еще раз подчеркнем, что перечисленные вьше восемь семейств не исчерпывают списка многообразий, не удовлетворяющих $K^{2}$-условию. Имеются еще семь семейств, не удовлетворяюших $K$-условию, большинство которых не является бирационально жесткими, обладая многими структурами рационально связного расслоения. Эти семейства в настоящей работе не рассматриваются.

0.3. Многообразия с пучком двойных пространств. Бирациональная жесткость многообразий $V$, расслоенных на двойные пространства индекса 1 , была доказана в самой первой работе автора, посвященной расслоениям Фано, в [2], в предположении достаточной закрученности по базе, т.е. $K^{2}$-условия. В настояшей статье будут рассмотрены многообразия этого типа, удовлетворяющие $K$-условию, но не $K^{2}$-условию. Напомним их конструкцию, являюшуюся упрощением приведенной выше конструкции для двойных гиперповерхностей (и соответствующую значению $m=1)$. Пусть $\mathscr{E}=\bigoplus \mathscr{O}_{\mathbb{P}^{1}}\left(a_{i}\right)$ - локально свободньй пучок ранга $M+1, a_{0}=0 \leqslant \cdots \leqslant a_{M}, X=\mathbb{P}(\mathscr{E})$ - его проективное расслоение, тогда $V$ реализуется как двойное накрытие $\sigma: V \rightarrow X$, разветвленное над гладкой гиперповерхностью $W \subset X$,

$$
W \sim 2 M L_{X}+2 a_{W} R,
$$

где $L_{X}-$ класс тавтологического пучка, $R$ - класс слоя проекции $\pi_{X}: X \rightarrow \mathbb{P}^{3}$. Очевидно,

$$
K_{V}=-L_{V}+\left(a_{X}+a_{W}-2\right) F,
$$

где $L_{V}=\sigma_{*} L_{X}, F=\sigma^{*} R$ - класс слоя,

$$
\text { Pic } V=\mathbb{Z} L_{V} \oplus \mathbb{Z} F
$$

$a_{X}=a_{1}+\cdots+a_{M}, a_{W} \in \mathbb{Z}_{+}$. Параметры семейства записываем в таком формате:

$$
\left(\left(a_{1}, \ldots, a_{M}\right), a_{W}\right),
$$


причем, как и вьшше, если $\left(a_{1}, \ldots, a_{M}\right) \neq(0, \ldots, 0)$, то нули опускаем, а если $\left(a_{1}, \ldots, a_{M}\right)=(0, \ldots, 0)$, то пишем просто $(0)$. Многообразие $V$ удовлетворяет $K^{2}$-условию, если выполнено неравенство

$$
\left(K_{V}^{2} \cdot L_{V}^{M-1}\right)=8-2 a_{X}-4 a_{W} \leqslant 0 .
$$

В настоящей работе рассматриваются семейства, не удовлетворяющие (2). Приведем их список:

$$
\begin{array}{ll}
1^{*} . & ((1), 1) ; \\
2^{*} . & ((2), 0) ; \\
3^{*} . & ((3), 0) ; \\
4^{*} . & ((1,2), 0) ; \\
5^{*} . & ((1,1,1), 0) .
\end{array}
$$

Для всех перечисленных типов многообразий $K$-условие выполнено (это будет показано ниже). Имеются еше четыре семейства, не удовлетворяющие $K^{2}$ - и $K$-условиям. Их бирациональная геометрия будет изучена в последующей работе.

0.4. Формулировка основного результата. Для дивизора $D$ на рационально связном многообразии $Y$ полагаем

$$
c(D, Y)=\sup \left\{\varepsilon \in \mathbb{Q}_{+} \mid D+\varepsilon K_{Y} \in A_{+}^{1} Y\right\} .
$$

Для подвижной линейной системы $\Sigma$ на унилинейчатом многообразии $V$ определим виртуальный порог канонического присоединения формулой

$$
c_{\text {virt }}(\Sigma)=\inf _{V \# \rightarrow V}\left\{c\left(\Sigma^{\#}, V^{\#}\right)\right\},
$$

где точная нижняя грань взята по всем бирациональньм морфизмам $V^{\#} \rightarrow V$, $V^{\#}$ - проективная модель поля $\mathbb{C}(V)$, неособая в коразмерности $1, \Sigma^{\#}-$ собственньй прообраз системы $\Sigma$ на $V^{\#}$. Следуюшее определение бирациональной жесткости эквивалентно стандартному.

ОПРЕДЕЛЕНИЕ 0.1. (i) Многообразие $V$ называется бирационально сверхжес$m \kappa u м$, если для любой подвижной линейной системы $\Sigma$ на $V$ имеет место равенство

$$
c_{\mathrm{virt}}(\Sigma)=c(\Sigma, V)
$$

(ii) Многообразие $V$ (соответственно расслоение $\Phi$ ано $V / S$ ) называется бирационально жместким, если для любой подвижной линейной системы $\Sigma$ на $V$ сушествует бирациональный автоморфизм $\chi \in \operatorname{Bir} V$ (соответственно послойный автоморфизм $\chi \in \operatorname{Bir}(V / S))$, обеспечивающий равенство

$$
c_{\mathrm{virt}}(\Sigma)=c\left(\chi_{*} \Sigma, V\right)
$$

Сформулируем основной результат работы. 
ТеОРема 1. (i) Регулярные многообразия $V$ с пучком двойныц гиперповерхностей типов 2-8 в приведенном выше списке и все многообразия типов $1^{*}-5^{*}$ с пучком двойньх пространств являются бирационально сверхжсесткими и удовлетворяют $K$-условию: $-K_{V} \notin A_{\mathrm{mov}}^{1} V$. Имеющаяся на этих многообразиях структура рационально связного расслоения $V / \mathbb{P}^{1}$ - единственная нетривиальная структура рационально связного расслоения на $V$. Группь бирациональных и бирегулярных автоморфизмов әтих многообразий совпадают:

$$
\operatorname{Bir} V=\text { Aut } V=\mathbb{Z} / 2 \mathbb{Z} \text {. }
$$

(ii) Регулярнье многообразия $V$ типа 1 (т.е. многообразия семейства $((0),(2,0)))$ являются бирационально жесткими. Они обладают нетривиальным бирациональным автоморфизмом - инволющией $\tau \in \operatorname{Bir} V \backslash$ Aut $V$ $u$ не удовлетворяют $K$-условию. На $V$ имеется ровно две нетривиальные структурь рационально связного расслоения: пучок $|F|$ слоев морфизма $\pi$ и его образ $\left|\tau_{*} F\right|$. Группа $\operatorname{Bir} V$ состоит из четырех элементов:

$$
\operatorname{Bir} V=\langle\tau\rangle \times \text { Aut } V=(\mathbb{Z} / 2 \mathbb{Z})^{\times 2} \text {. }
$$

СЛЕДСТВИЕ 0.1. Все многообразия типов 1-8 и $1^{*}-5^{*}$ не имеют структур рачионально связного расслоения над базой размерности 2 или выше, в частности, не могут быть расслоены рациональным отображсением на рациональные кривые или рациональные поверхности. Тем самым, все они нерациональны.

0.5. Структура статьи. Для доказательства теоремы 1 необходимо усилить технику метода максимальных особенностей. Такое усиление важно само по себе, так как расширяет область применимости метода: еще далеко не для всех естественных семейств расслоений Фано над $\mathbb{P}^{1}$ доказана бирациональная жесткость, даже при условии достаточной закрученности по базе. Поэтому большая часть статьи носит технический характер.

В $\S 1$ проведена подготовительная работа: совпадение виртуального и фактического порогов канонического присоединения доказано в предположении, что выполнено $K^{2}$-условие и слои расслоения $\Phi$ ано $V / \mathbb{P}^{1}$ удовлетворяют стандартньп условиям (h), (v) и (vs) (cм. [1]). Затем сделан первый шаг в направлении усиления техники: показано, что усиление условия (h) компенсирует ослабление $K^{2}$-условия.

Параграфф 2 посвяшен усилению техники метода максимальных особенностей на основе совершенно другой идеи: сопоставления особенностей горизонтального цикла $Z^{h}$ и его ограничения на слой $F$, содержащий центр максимальной особенности. Эта идея является новой и используется для доказательства бирациональной жесткости впервые. Кроме того, приводится со всеми подробностями техника подсчета кратностей: во всех предшествовавших работах [1]-[10] вычислялась кратность цикла $Z$ - пересечения двух дивизоров линейной системы $\Sigma$, здесь же речь идет о пересечении дивизора и неприводимого подмногообразия коразмерности 2. Вычисления параллельны дивизориальному случаю, однако имеются некоторые различия: например, учитываются только раздутия подмногообразий коразмерности 3 и выше, причем раздутия подмногообразий коразмерности 3 играют ту же роль, что в дивизориальном случае - коразмерности 2. 
В $\S 3$ доказывается теорема 1 . Сначала изучен вопрос о справедливости $K$-условия для многообразий из приведенных выше списков. Затем проверены необходимые оценки для подмногообразий $Y \subset F$ произвольного слоя $F$ (большая часть этой работы проделана в предыдушей статье [1] и в [4]). Оставшаяся часть параграфа посвящена усилению оценки на кратности подмногообразий коразмерности два, для чего, в свою очередь, необходимо оценить количество прямых (с учетом их кратностей), проходящих через произвольную точку слоя. Комбинация этой усиленной оценки с общей теорией, развитой в первых двух параграфах, позволяет доказывать совпадение виртуального и фактического порогов канонического присоединения для многообразий, еще сильнее отклоняющихся от $K^{2}$-условия, чем многообразия, рассматриваемые в настоящей работе. Эти предельные возможности техники метода максимальных особенностей будут использованы в дальнейшем.

0.6. Исторические замечания. Сразу же после того, как в [2] была развита эффективно работаюшая техника изучения бирациональной геометрии расслоений Фано $V / \mathbb{P}^{1}$, удовлетворяющих $K^{2}$-условию, на повестку дня встал вопрос об изучении "пограничных" классов, для которых отклонение от $K^{2}$-условия не слишком велико. Уже в [3] отмечено, что для конкретных изученных многообразий $K^{2}$-условие излишне сильное: неравенства, с помощью которых устанавливается бирациональная жесткость, содержат значительный "запас". Поэтому некоторое ослабление этого условия не должно влиять на окончательный результат. Работы М.М. Гриненко [11]-[13] и И.В. Соболева [14], [15] подтвердили данное предположение. В [12] высказана гипотеза, что $K$-условие (более слабое, чем $K^{2}$-условие) уже является достаточным для бирациональной жесткости. Эта гипотеза недавно вызвала интерес [16] в связи с попытками изучать бирациональную геометрию трехмерных расслоений на коники (над $\left.\mathbb{P}^{2}\right)$, не удовлетворяюших условию Саркисова [17], [18] (это условие является точным аналогом $K^{2}$-условия). Однако в работах [14], [15] успешно изучены многообразия, не удовлетворяюшие и $K$-условию (хотя, конечно, отклоняюшиеся от него не слишком сильно). При этом в [14], [15] использовалась техника существенно более слабая, чем доступная сегодня (§2 настоящей статьи). Все это свидетельствует о том, что механизмы, контролирующие бирациональную жесткость (может быть, следовало бы говорить о "степени бирациональной жесткости"), до сих пор неизвестны. С другой стороны, имеются основания для оптимизма в отношении перспектив изучения бирациональной геометрии расслоений Фано методом максимальных особенностей.

0.7. Благодарности. Сушественная часть настояшей работы проделана автором в Институте Макса Планка в Бонне осеню 2003 г. Автор благодарит Математический институт Макса Планка в Бонне за гостеприимство и прекрасные условия работы.

\section{§1. Метод максимальных особенностей}

В $\S 1$ напоминаются основные факты метода максимальных особенностей: неравенство Нётера-Фано, понятия максимальных и сверхмаксимальных особенностей, вычисление самопересечения подвижной линейной системы [5], [8], [10]. Доказана теорема 2 предшествуюшей работы [1]. Затем техника модифицирована с учетом отклонения от $K^{2}$-условия. 


\section{1. Максимальные особенности линейных систем. Пусть}

$$
\Sigma \subset\left|-n K_{V}+l F\right|, \quad l \in \mathbb{Z}_{+},
$$

- подвижная линейная система на многообразии $V$. Поскольку $l \geqslant 0$, имеем

$$
c(\Sigma)=n,
$$

причем $n=0$ тогда и только тогда, когда линейная система $\Sigma$ составлена из пучка $|F|$, т.е. поднята с базы $\mathbb{P}^{1}$.

ЗАмЕчАниЕ 1.1. Если расслоение $V / \mathbb{P}^{1}$ удовлетворяет $K^{2}$-условию:

$$
K_{V}^{2} \notin \operatorname{Int} A_{+}^{2} V
$$

то для любой подвижной системы $\Sigma \subset\left|-n K_{V}+l F\right|$ имеем $l \geqslant 0$. В самом деле, самопересечение линейной системы $\Sigma$

$$
\left(-n K_{V}+l F\right)^{2}=n^{2} K_{V}^{2}+2 n l H_{F}
$$

есть класс эффективного цикла коразмерности два. В силу $K^{2}$-условия отсюда вытекает, что $l \in \mathbb{Z}_{+}$.

Предположим, что имеет место неравенство

$$
c_{\text {virt }}(\Sigma)<c(\Sigma)=n
$$

т.е. существует модель $V^{\#}$ многообразия $V$ такая, что

$$
c\left(\Sigma^{\#}, V^{\#}\right)<c(\Sigma, V) .
$$

ПРЕДЛОЖЕнИЕ 1.1. Существует простой дивизор $E \subset V^{\#}$, для которого выполнено неравенство Нётера-Фано

$$
\nu_{E}(\Sigma)>n \cdot a(V, E)
$$

Геометрическое дискретное нормирование $\nu_{E}$ поля рациональных функций $\mathbb{C}(V)$ или любой простой дивизор $E^{+} \subset V^{+}$на любой модели $V^{+}$многообразия $V$, peализующий это дискретное нормирование, назьвается максимальной особенностью линейной системы $\Sigma$.

По поводу доказательства предложения 1.1 см. любую из работ [2], [5], [8], [10], [19].

Отметим, что $a(V, E)$ в $(3)$ обозначает дискрепантность $E$ относительно исходной модели $V$. Таким образом, лог-пара

$$
\left(V, \frac{1}{n} \Sigma\right)
$$

не канонична: исключительный дивизор $E$ реализует ее особенность, не являющуюся канонической. 
Поскольку линейная система $\Sigma$ подвижна, а $\nu_{E}(\Sigma)>0$, центр дискретного нормирования $\nu_{E}$ на $V$ есть подмногообразие $B=\operatorname{centre~}_{V}\left(\nu_{E}\right) \subset V$ коразмерности не меньше двух. Пусть

$$
\begin{aligned}
\varphi_{i, i-1}: & V_{i} \rightarrow V_{i-1} \\
\cup & \cup \\
E_{i} & \rightarrow B_{i-1}
\end{aligned}
$$

- последовательность раздутий с неприводимыми центрами $B_{i-1} \subset V_{i-1}$, однозначно определенная условиями

1) $V_{0}=V, B_{0}=B, i=1, \ldots, K$

2) $B_{j}=\operatorname{centre}_{V_{j}}\left(\nu_{E}\right) \subset V_{j}, E_{j+1}=\varphi_{j+1, j}^{-1}\left(B_{j}\right)$;

3) нормирование $\nu_{E_{K}}$ совпадает с $\nu_{E}$.

Иными словами, бирациональное отображение

$$
V_{K} \rightarrow V^{\#}
$$

бирегулярно в общей точке дивизора $E_{K}$ и преобразует $E_{K}$ в $E$. Символом $\Sigma^{j}$ обозначим собственный прообраз линейной системы $\Sigma$ на $V_{j}$. Положим

$$
\nu_{j}=\operatorname{mult}_{B_{j-1}} \Sigma^{j-1}, \quad \delta_{j}=\operatorname{codim} B_{j-1}-1 .
$$

На множестве исключительных дивизоров

$$
\left\{E_{1}, \ldots, E_{K}\right\}
$$

зададим обычным образом [5], [8], [19] структуру ориентированного графа: $E_{i}$ и $E_{j}$ соединены ориентированным ребром, если и только если $i>j$ и

$$
B_{i-1} \subset E_{j}^{i-1},
$$

обозначение: $i \rightarrow j$. Как обычно, пусть при $i>j$

$$
p_{i j}=\#\left\{\text { пути из } E_{i} \text { в } E_{j}\right\} \geqslant 1,
$$

$p_{i i}=1$ по определению. Положим $p_{i}=p_{K i}$. Неравенство Нётера-Фано принимает традиционный вид

$$
\sum_{i=1}^{K} p_{i} \nu_{i}>n \sum_{i=1}^{K} p_{i} \delta_{i} .
$$

1.2. Усиление неравенства Нётера-Фано. Предположим теперь, что общий слой $F=F_{t}$ расслоения $V / \mathbb{P}^{1}$ не допускает подвижных линейных систем, обладающих максимальной особенностью, т.е. для любой подвижной системы $\Sigma_{F} \subset$ $\left|n H_{F}\right|=\left|-n K_{F}\right|$ и любого геометрического дискретного нормирования $\nu_{E^{*}}$ имеет место неравенство

$$
\nu_{E^{*}}\left(\Sigma_{F}\right) \leqslant n a\left(E^{*}\right) .
$$

ПРЕДЛОЖЕНИЕ 1.2. Центр В максимальной особенности $\nu_{E}$ на $V$ содержится в некотором слое $\pi^{-1}(t)=F_{t}, t \in \mathbb{P}^{1}$. 
ДокАЗАТЕЛЬСТво. Предположим противное: $\pi(B)=\mathbb{P}^{1}$. Пусть $F \subset V$ - слой общего положения. Легко видеть, что ограничение $\Sigma_{F}=\left.\Sigma\right|_{F}$ линейной системы $\Sigma$ на $F$ есть подвижная линейная система $\Sigma_{F} \subset\left|n H_{F}\right|$, обладающая максимальной особенностью $\nu_{E^{*}}=\left.\nu_{E}\right|_{F}$, которую проще всего определить, ограничивая последовательность раздутий (4) на слой $F$ и замечая, что дискрепантность остается той же самой:

$$
a\left(\left.E\right|_{F}, F\right)=a(E, V),
$$

именно потому, что $B$ накрывает базу. Центр нормирования $\left.\nu_{E}\right|_{F}$ есть $B_{F}=$ $B \cap F$. Однако это противоречит сделанному выше предположению. Следовательно, $\pi(B) \neq \mathbb{P}^{1}$. Предложение доказано.

Пусть $\mathscr{M}=\left\{T_{1}, \ldots, T_{k}\right\}$ - множество всех простых дивизоров на $V^{\#}$, удовлетворяющих неравенству Нётера-Фано (см. предложение 1.1). Как было только что доказано, центр $B_{E}=\operatorname{centre}\left(\nu_{E}\right)$ любой максимальной особенности $E \in \mathscr{M}$ содержится в некотором слое $F_{t}$. Множество $\mathscr{M}$ конечно (потому что фиксирована модель $\left.V^{\#}\right)$, так что существует не более чем конечное множество точек $t \in \mathbb{P}^{1}$, слои над которыми $F_{t}$ содержат центры максимальных особенностей. Положим $\mathscr{M}_{t}=\left\{E \in \mathscr{M} \mid B_{E} \subset F_{t}\right\}$,

$$
e(E)=\nu_{E}(\Sigma)-n a(E, V)>0
$$

для $E \in \mathscr{M}$. Напомним, что $\Sigma \subset\left|-n K_{V}+l F\right|, l \in \mathbb{Z}_{+}$.

ПРЕДЛОЖЕНИЕ 1.3. Имеет место неравенство

$$
\sum_{t \in \mathbb{P}^{1}} \max _{\left\{E \in \mathscr{M}_{t}\right\}} \frac{e(E)}{\nu_{E}\left(F_{t}\right)}>l
$$

ДокАЗАТЕЛЬСтво. Пусть $D^{\#} \in \Sigma^{\#}$ - общий дивизор, т.е. собственный прообраз некоторого дивизора $D \in \Sigma$ общего положения на $V$ \#. По предположению линейная система

$$
\left|D^{\#}+n K_{V \#}\right|
$$

пуста. Поэтому пуста линейная система

$$
\left|l F-\sum_{E \in \mathscr{M}} e(E) E\right| .
$$

С другой стороны, для $E \in \mathscr{M}_{t}$ по построению дивизор

$$
F_{t}-\nu_{E}\left(F_{t}\right) E
$$

эффективен, так что эффективен и дивизор

$$
\sum_{t \in \mathbb{P}_{1}}\left[\left(\max _{\left\{E \in \mathscr{M}_{t}\right\}} \frac{e(E)}{\nu_{E}\left(F_{T}\right)}\right) F_{t}-\sum_{E \in \mathscr{M}_{t}} e(E) E\right] .
$$

Отсюда немедленно получаем неравенство (5). Предложение доказано. 
1.3. Самопересечение линейной системы $\Sigma$. Пусть $D_{i} \in \Sigma, i=1,2,-$ обшие дивизоры, так что замкнутое множество $D_{1} \cap D_{2}$ имеет коразмерность два. Пусть

$$
Z=\left(D_{1} \circ D_{2}\right)=Z^{v}+Z^{h}
$$

- разложение алгебраического цикла теоретико-схемного пересечения этих дивизоров на вертикальную $\left(Z^{v}\right)$ и горизонтальную $\left(Z^{h}\right)$ части. Для цикла $Z^{v}$ имеем дальнейшее разложение

$$
Z^{v}=\sum_{t \in \mathbb{P}^{1}} Z_{t}^{v}, \quad \operatorname{Supp} Z_{t}^{v} \subset F_{t} .
$$

Пусть $E \in \mathscr{M}_{t} \subset \mathscr{M}$ - некоторая максимальная особенность. Зафиксируем $t$ и $E$ и применим к циклу $Z_{t}^{v}+Z^{h}$ технику подсчета кратностей, развитую в [3], [5], [8]. До конца этого пункта $t$ и $E$ фиксированы, так что для упрошения обозначений пишем далее $F, Z^{v}, e, B$ вместо $F_{t}, Z_{T}^{v}, e(E), B_{E}=\operatorname{centre}(E)$ соответственно.

Лемма 1.1. Имеет место неравенство

$$
\operatorname{codim}_{F} B \geqslant 2 \text {. }
$$

ДоКАЗАТЕЛЬСТво. Предположим противное: $B \subset F$-простой дивизор. Пусть $D \in \Sigma$ - общий дивизор, $D_{F}$ - его ограничение на $F$. В силу неравенства НётераФано mult $_{B} D>n$, так что

$$
D_{F}=\alpha B+D^{\#},
$$

где $\alpha>n$ и $D^{\#}$ - эффективный дивизор на $F$. Однако

$$
D_{F} \sim n H_{F},
$$

что сразу дает противоречие. Лемма доказана.

Рассмотрим последовательность раздутий (4), связанную с дискретным нормированием $E$. Мы пользуемся обозначениями п. 1.1. Собственные прообразы $\left(Z^{h}\right)^{j},\left(Z^{v}\right)^{j}$ на $V_{j}$ циклов $Z^{h}, Z^{v}$ корректно определены. Символом $F^{j}$ обозначим собственный прообраз слоя $F$ на $V_{j}$. Положим

$$
N=\max \left\{i \mid B_{i-1} \subset F^{i-1}\right\} .
$$

Нетрудно видеть, что $\varphi_{i, i-1}\left(B_{i}\right)=B_{i-1}$ для любого $i=1, \ldots, K-1$, так что коразмерности $\operatorname{codim} B_{i}$ не возрастают. Положим

$$
L=\max \left\{i \mid \operatorname{codim} B_{i-1} \geqslant 3\right\} \leqslant K .
$$

Введем следующие обозначения: при $i \in\{1, \ldots, L\}$

$$
m_{i}^{h}=\operatorname{mult}_{B_{i-1}}\left(Z^{h}\right)^{i-1}, \quad m_{i}^{v}=\operatorname{mult}_{B_{i-1}}\left(Z^{v}\right)^{i-1},
$$

$m_{i}^{h(v)} \leqslant m_{i-1}^{h(v)}$ для $i=2, \ldots, L$.

Положим также

$$
\mu_{i}=\text { mult }_{B_{i-1}} F^{i-1} .
$$

Очевидно, $\mu_{i}=0$ при $i \geqslant N+1$. Тем более, $m_{i}^{v}=0$ при $i \geqslant N+1$ (если $N<L)$. Обозначая, как вьше, символом $p_{i}$ число путей в графе $\Gamma$ разрешения (4) из вершины $E=E_{K}$ в $E_{i}$, получаем 
ПРЕДЛОЖЕНИЕ 1.4. Имеет место неравенство

$$
\sum_{i=1}^{L} p_{i} m_{i}^{h}+\sum_{i=1}^{\min \{N, L\}} p_{i} m_{i}^{v} \geqslant \sum_{i=1}^{K} p_{i} \nu_{i}^{2} \geqslant \frac{\left(n \sum_{i=1}^{K} p_{i} \delta_{i}+e\right)^{2}}{\sum_{i=1}^{K} p_{i}} .
$$

ДоКАЗАТЕЛьСТво получается применением техники подсчета кратностей [3], [5], [8] в сочетании с условием

$$
\sum_{i=1}^{K} p_{i} \nu_{i}=n \sum_{i=1}^{K} p_{i} \delta_{i}+e
$$

$e>0$. Здесь мы не будем повторять этих стандартных рассуждений, отсылая читателя к [3].

1.4. $K^{2}$-условие и бирациональная жесткость. В качестве первого примера использования описанной выше техники докажем теорему 2 предшествующей работы [1]. Приводимые рассуждения повторяют данное в [2], [3] доказательство частных случаев этой теоремы, относящихся к конкретным семействам расслоений Фано. Здесь мы рассматриваем общий случай.

Предположим, что расслоение $V / \mathbb{P}^{1}$ удовлетворяет $K^{2}$-условию:

$$
K_{V}^{2} \notin \operatorname{Int} A_{+}^{2} V .
$$

Тогда для некоторого $\alpha \in \mathbb{Z}_{+}$имеет место соотношение

$$
Z^{h} \sim n^{2} K_{V}^{2}+\alpha H_{F}
$$

так что

$$
\operatorname{deg} Z^{v}=\sum_{t \in \mathbb{P}^{1}} \operatorname{deg} Z_{t}^{v} \leqslant(2 n \operatorname{deg} V) l .
$$

ПРЕДЛОЖЕНИЕ 1.5. Для некоторого $t \in \mathbb{P}^{1}$ существует максимальная особенность $E \in \mathscr{M}_{t}$, удовлетворяющая оценке

$$
e(E)>\frac{\nu_{E}\left(F_{t}\right)}{2 n \operatorname{deg} V} \operatorname{deg} Z_{t}^{v} .
$$

Следуя [2], [3], назовем особенность $E$, удовлетворяющую неравенству (8), сверхмаксимальной.

Для доказательства предложения 1.5 достаточно сравнить неравенства (5) и (7).

Теперь в обозначениях п. 1.3 положим

$$
\Sigma_{l}=\sum_{i=1}^{L} p_{i}, \quad \Sigma_{u}=\sum_{i=L+1}^{K} p_{i}, \quad \Sigma_{f}=\sum_{i=2}^{\min \{N, L\}} p_{i} .
$$

Отметим, что

$$
\nu_{E}(F)=\sum_{i=1}^{N} p_{i} \mu_{i} \leqslant p_{1} \mu_{1}+\mu_{2} \Sigma_{f}
$$


по определению кратностей $\mu_{i}$. Очевидно,

$$
m_{i}^{h} \leqslant m_{h}=m_{1}^{h}=\operatorname{mult}_{B} Z^{h}
$$

Положим также

$$
d_{h}=\operatorname{deg} Z^{h}, \quad d_{v}=\operatorname{deg} Z_{t}^{v}
$$

и введем коэффициенты

$$
k_{h}=\frac{m_{h}}{d_{h}} \operatorname{deg} V, \quad k_{v}=\frac{\operatorname{deg} V}{\nu_{E}(F) d_{v}} \sum_{i=1}^{\min \{N, L\}} p_{i} m_{i}^{v}
$$

Из предложения 1.5 вытекает

СлЕДСТВИЕ 1.1. Имеет место неравенство

$$
\left(4-k_{h}\right) \Sigma_{l}\left(\Sigma_{l}+\Sigma_{u}\right) n^{2}+\Sigma_{u}^{2} n+e^{2}+2\left(2-k_{v}\right) \Sigma_{l} n e+2\left(1-k_{v}\right) \Sigma_{u} n e<0 .
$$

ДоказАтЕльство. В неравенстве (6) заменим $m_{i}^{h}$ на $m_{h}=k_{h} d_{h} / \operatorname{deg} V=$ $k_{h} n^{2}$, числа $\delta_{i}$ при $i \leqslant L-$ на 2 , отчего неравенство может лишь усилиться, и с учетом определения коэффициента $k_{v}$ после несложных преобразований получим

$$
\left(4-k_{h}\right) \Sigma_{l}\left(\Sigma_{l}+\Sigma_{u}\right) n^{2}+\left(n \Sigma_{u}+e\right)^{2}+4 \Sigma_{l} e n-k_{v} \frac{d_{v} \nu_{E}(F)}{\operatorname{deg} V}\left(\Sigma_{l}+\Sigma_{u}\right) \leqslant 0 .
$$

Учитывая определение сверхмаксимальной особенности (предложение 1.5), заменим $d_{v} \nu_{E}(F)$ на $2 n e \operatorname{deg} V$. При этом неравенство станет строгим и мы получим в точности то, что утверждалось. Следствие доказано.

Предположим теперь, что расслоение $V / \mathbb{P}^{1}$ удовлетворяет

- условию (v) работы [1], т.е. для любого неприводимого вертикального подмногообразия $Y$ коразмерности $2, Y \subset \pi^{-1}(t)=F_{t}$, и гладкой точки $o \in F_{t}$ выполнено неравенство

$$
\frac{\text { mult }_{o}}{\operatorname{deg}} Y \leqslant \frac{2}{\operatorname{deg} V}
$$

- условию (vs) работы [1], т.е. для любого вертикального подмногообразия $Y \subset F_{t}$ коразмерности 2 (относительно $V$, т.е. простого дивизора на $F_{t}$ ), особой точки $o \in F_{t}$ и бесконечно близкой точки $x \in \widetilde{F}_{t}$, где $\varphi: \widetilde{F}_{t} \rightarrow F_{t}-$ раздутие точки $o, \varphi(x)=o, \widetilde{Y} \subset \widetilde{F}_{t}$ - собственный прообраз подмногообразия $Y$ на $\widetilde{F}_{t}$, выполнены оценки

$$
\frac{\text { mult }_{o}}{\operatorname{deg}} Y \leqslant \frac{4}{\operatorname{deg} V}, \quad \frac{\operatorname{mult}_{x} \tilde{Y}}{\operatorname{deg} Y} \leqslant \frac{2}{\operatorname{deg} V}
$$

- условию (h) работы [1], т.е. для любого горизонтального подмногообразия $Y$ коразмерности 2 и точки $o \in Y$ выполнена оценка

$$
\frac{\text { mult }_{o}}{\operatorname{deg}} Y \leqslant \frac{4}{\operatorname{deg} V}
$$

В этих предположениях справедлива 
ЛЕмма 1.2. Имеют место оценки: $k_{h} \leqslant 4, k_{v} \leqslant 2$.

ДокАЗАТЕльство. Первое из этих двух неравенств вытекает непосредственно из определения числа $k_{h}$ и условия $(\mathrm{h})$, которому по предположению удовлетворяет расслоение $V / \mathbb{P}^{1}$. Докажем второе неравенство. Имеем

$$
\left(\frac{d_{v}}{\operatorname{deg} V}\right) k_{v}=\frac{\sum_{i=1}^{\min \{N, L\}} p_{i} m_{i}^{v}}{\sum_{i=1}^{N} p_{i} \mu_{i}} \leqslant \frac{p_{1} m_{1}^{v}+\Sigma_{f} m_{2}^{v}}{p_{1} \mu_{1}+\Sigma_{f}} .
$$

Если $\operatorname{dim} B \geqslant 1$ или $B=o \in F-$ неособая точка слоя, то $\mu_{1}=\cdots=\mu_{N}=1$ и в силу условия (v)

$$
m_{2}^{v} \leqslant m_{1}^{v} \leqslant 2 \frac{d_{v}}{\operatorname{deg} V}
$$

откуда сразу следует неравенство $k_{v} \leqslant 2$. Если $B=o \in F$ - особая точка слоя, то $\mu_{1} \geqslant 2$ и в силу условия (vs)

$$
m_{1}^{v} \leqslant \frac{4 d_{v}}{\operatorname{deg} V}, \quad m_{2}^{v} \leqslant \frac{2 d_{v}}{\operatorname{deg} V},
$$

откуда снова получаем, что $k_{v} \leqslant 2$. Лемма доказана.

Напомним формулировку теоремы 2 работы [1]:

Предположим, что гладкое стандартное расслоение Фано $V / \mathbb{P}^{1}$ удовлетворяет $K^{2}$-условию и условиям (v), (vs) и (h). Тогда $V / \mathbb{P}^{1}-$ бирачионально сверхжесткое расслоение Фано.

Завершим ее доказательство. Предположим, что она неверна. Тогда из следствия 1.1 и леммы 1.2 вытекает неравенство

$$
\Sigma_{u}^{2} n^{2}-2 \Sigma_{u} n e+e^{2}<0
$$

Такого не может быть. Противоречие. Теорема 2 работы [1] доказана.

1.5. Обобщенное $K^{2}$-условие. Развитые в [2], [3] и воспроизведенные выше в общем виде методы сохраняют силу для расслоений $\Phi$ ано $V / \mathbb{P}^{1}$, не удовлетворяющих $K^{2}$-условию. Если отклонение от $K^{2}$-условия не слишком велико, то техника п. 1.4 продолжает быть эффективной и требует лишь небольшой модификации. Прежде всего необходимо измерить отклонение от $K^{2}$-условия.

ОПРЕДЕЛЕНИЕ 1.1. Стандартное расслоение Фано $V / \mathbb{P}^{1}$ удовлетворяет обобщенному $K^{2}$-условию глубины $\varepsilon \geqslant 0$, если

$$
K_{V}^{2}-\varepsilon H_{F} \notin \operatorname{Int} A_{+}^{2} V .
$$

Как отмечалось в [3], для эффективных циклов коразмерности 2 на естественных многообразиях Фано справедливы более сильные оценки на кратности, чем этого требует доказательство бирациональной жесткости. Однако для расслоений Фано, не удовлетворяющих $K^{2}$-условию, эти усиленные оценки оказываются востребованньми. 
ОПРЕДЕЛЕНИЕ 1.2. Стандартное расслоение Фано $V / \mathbb{P}^{1}$ удовлетворяет обобщенному условию (h) глубины $\delta \geqslant 0$, если для любого горизонтального подмногообразия $Y \subset V$ коразмерности два и произвольной точки $o \in Y$ выполнено неравенство

$$
\frac{\operatorname{mult}_{o}}{\operatorname{deg}} Y \leqslant \frac{4-\delta}{\operatorname{deg} V}
$$

Начиная с этого момента и до конца параграфа предполагаем, что расслоение $V / \mathbb{P}^{1}$ удовлетворяет обобщенному $K^{2}$-условию глубины $\varepsilon \geqslant 0$ и обобщенному условию (h) глубины $\delta \geqslant 0$. Кроме того, предполагаются вьполненными условия (v) и (vs) (в прежней форме). Зафиксируем подвижную линейную систему $\Sigma \subset\left|-n K_{V}+l F\right| c l \in \mathbb{Z}_{+}$и предположим, что $c_{\mathrm{virt}}(\Sigma)<n$. Для горизонтальной части самопересечения линейной системы $\Sigma$ имеем

$$
Z^{h} \sim n^{2} K_{V}^{2}+\alpha H_{F}
$$

где коэффициент $\alpha \in \mathbb{Z}$ удовлетворяет неравенству

$$
\alpha \geqslant-\varepsilon n^{2} .
$$

Следовательно, для вертикальной компоненты имеем

$$
Z^{v} \sim(2 n l-\alpha) H_{F},
$$

откуда

$$
\operatorname{deg} Z^{v}=\sum_{t \in \mathbb{P}^{1}} \operatorname{deg} Z_{t}^{v} \leqslant\left(2 n l+\varepsilon n^{2}\right) \operatorname{deg} V .
$$

ПрЕДЛОЖЕнИЕ 1.6. Для некоторой точки $t \in \mathbb{P}^{1}$ существует максимальная особенность $E \in \mathscr{M}_{t} \neq \varnothing$, удовлетворяющая оценке

$$
e(E)>\frac{\nu_{E}\left(F_{t}\right)}{2}\left(\frac{\operatorname{deg} Z_{t}^{v}}{n \operatorname{deg} V}-\varepsilon n\right)
$$

ЗАмечАниЕ 1.2 . При $\varepsilon=0$ получаем предложение 1.5 .

ДоКАЗАТЕЛЬСТво ПРЕДЛоЖЕНИЯ 1.6. Сравним неравенства (5) и (10). Заменяя число $l$ в правой части неравенства (10) левой частью неравенства $(5)$, получаем

$$
\sum_{t \in \mathbb{P}^{1}}\left[\operatorname{deg} Z_{t}^{v}-2 n \operatorname{deg} V \max _{\left\{E \in \mathscr{M}_{t}\right\}} \frac{e(E)}{\nu_{E}\left(F_{t}\right)}\right]<\varepsilon n^{2} \operatorname{deg} V,
$$

откуда немедленно следует наше предложение.

ЗАмЕчАниЕ 1.3. Если имеется несколько максимальных особенностей, центры которых лежат в слоях над различными точками $t_{1}, \ldots, t_{k}$, то предложение 1.6 усиливается: существует максимальная особенность $E \in \mathscr{M}_{t}, t \in\left\{t_{1}, \ldots, t_{k}\right\}$, удовлетворяюшая оценке

$$
e(E)>\frac{\nu_{E}\left(F_{t}\right)}{2}\left(\frac{\operatorname{deg} Z_{t}^{v}}{n \operatorname{deg} V}-\frac{\varepsilon n}{k}\right) .
$$


Впрочем, такое усиление мало что дает, так как для доказательства бирациональной жесткости, т.е. полной реализации схемы метода максимальных особенностей, нужно рассматривать наихудшую возможность.

Теперь рассуждаем как при доказательстве следствия 1.1 с единственной разницей: выражение $d_{v} \nu_{E}(F)$ заменяем в неравенстве $(9)$ на выражение

$$
\left(2 n e+\varepsilon n^{2} \nu_{E}(F)\right) \operatorname{deg} V
$$

при этом неравенство становится строгим. Учитьвая, что условия (h), (v) и (vs) по-прежнему выполнены, так что лемма 1.2 верна, получаем неравенство

$$
\left(\left(4-k_{h}\right) \Sigma_{l}-k_{v} \varepsilon \nu_{E}(F)\right)\left(\Sigma_{l}+\Sigma_{u}\right) n^{2}+\left(n \Sigma_{u}-e\right)^{2}<0 .
$$

По определению числа $k_{v}$ имеем

$$
k_{v} \nu_{E}(F)=\operatorname{deg} V \sum_{i=1}^{\min \{N, L\}} p_{i} \frac{m_{i}^{v}}{d_{v}} \leqslant 2 \Sigma_{l} .
$$

Следовательно, имеет место неравенство

$$
\left(4-k_{h}-2 \varepsilon\right) \Sigma_{l}\left(\Sigma_{l}+\Sigma_{u}\right) n^{2}+\left(n \Sigma_{u}-e\right)^{2}<0 .
$$

Вспомним, что в силу обобщенного условия (h) глубины $\delta \geqslant 0$ для коэффициента $k_{h}$ справедлива оценка

$$
k_{h} \leqslant 4-\delta .
$$

Отсюда немедленно вытекает

ПРЕДЛОЖЕНИЕ 1.7. Если $\delta \geqslant 2 \varepsilon$, то имеет место равенство

$$
c_{\mathrm{virt}}(\Sigma)=c(\Sigma)=n .
$$

В частности, если для любой подвижной линейной системы $\Sigma \subset\left|-n K_{V}+l F\right|$ имеем $l \in \mathbb{Z}_{+}$, то расслоение $V / \mathbb{P}^{1}$ бирационально сверхэсесткое.

\section{§2. Усиленная техника подсчета кратностей}

Цель настоящего параграфа - усилить неравенство (12) и предложение 1.7. Для этого используются соображения размерности раздуваемых подмногообразий $B_{i-1}$ : чем меньше размерность раздуваемого подмногообразия, тем выше дискрепантность исключительного дивизора и, следовательно, тем сильнее оценка на кратность $\nu_{E}(\Sigma)$. С другой стороны, единственный реальный способ оценить сверху особенности горизонтального цикла $Z^{h}$ заключается в том, чтобы ограничить $Z^{h}$ на слой $F$ и оценить особенности эффективного цикла $\left(Z^{h} \circ F\right)$ коразмерности два на $F$. Этим способом мы пользовались выше; и в работах [1]-[3] особенности цикла $Z^{h}$ оценивались именно таким образом. При этом до сих пор никогда не учитывался вклад подмногообразий $B_{i-1}$ коразмерности три (относительно $V$ ), содержащихся в собственном прообразе $F^{i-1}$ слоя $F$ (если таковые существуют). Именно это и сделано ниже. В результате получены оценки, позволяющие исключить максимальную особенность даже при существенном отклонении от $K^{2}$-условия: достаточно, если выполнено обобщенное $K^{2}$-условие глубины 2. 
2.1. Обозначения и формулировка основного утверждения. Продолжаем изучать подвижную линейную систему $\Sigma \subset\left|-n K_{V}+l F\right|, l \in \mathbb{Z}_{+}$, удовлетворяюшую неравенству

$$
c_{\text {virt }}(\Sigma)<c(\Sigma)=n
$$

Все обозначения $\S 1$ сохраняют силу. Однако если в пा. 1.4 и 1.5 мы объединяли раздутия всех подмногообразий коразмерности 3 и выше, то теперь будем рассуждать тоньше. Положим

$$
\begin{aligned}
J_{s} & =\left\{i \mid 1 \leqslant i \leqslant K, \operatorname{codim} B_{i-1} \geqslant 4\right\}, \\
J_{m} & =\left\{i \mid 1 \leqslant i \leqslant K, \operatorname{codim} B_{i-1}=3\right\}, \\
J_{u} & =\{i \mid L+1 \leqslant i \leqslant K\}, \quad J_{l}=J_{s} \cup J_{m} .
\end{aligned}
$$

В свою очередь, разобьем множество $J_{m}$ на два непересекающихся подмножества, $J_{m}=J_{m}^{+} \sqcup J_{m}^{-}$где

$$
J_{m}^{+}=\left\{i \in J_{m} \mid B_{i-1} \subset F^{i-1}\right\},
$$

$J_{m}^{-}=J_{m} \backslash J_{m}^{+}=\left\{i \in J_{m} \mid B_{i-1} \not \subset F^{i-1}\right\}$. Может оказаться, что $J_{m}^{+}$или $J_{m}^{-}$(или все множество $J_{m}$ ) пусто. Положим, далее,

$$
\Sigma_{s}=\sum_{i \in J_{s}} p_{i}, \quad \Sigma_{m}^{ \pm}=\sum_{i \in J_{m}^{ \pm}} p_{i}, \quad \Sigma_{m}=\Sigma_{m}^{+}+\Sigma_{m}^{-},
$$

символ $\Sigma_{u}$ сохраняет прежний смысл. В обозначениях п. 1.4 имеем $\Sigma_{l}=\Sigma_{s}+\Sigma_{m}$.

Теперь неравенство (6) можно переписать в виде

$$
\sum_{i \in J_{l}} p_{i} m_{i}^{h}+\sum_{i \in J_{S} \cup J_{m}^{+}} p_{i} m_{i}^{v} \geqslant \frac{\left(\left(3 \Sigma_{s}+2 \Sigma_{m}+\Sigma_{u}\right) n+e\right)^{2}}{\Sigma_{s}+\Sigma_{m}+\Sigma_{u}} .
$$

Напомним, что $\mu_{i}=\operatorname{mult}_{B_{i-1}} F^{i-1}, \mu_{i}=1$ при $i \geqslant 2$, и для $\mu_{1}$ имеются две возможности: $\mu_{1}=1$ или $\mu_{1}=2$.

ПРЕДЛОЖЕНИЕ 2.1. Имеет место оценка

$$
\sum_{i \in J_{s} \cup J_{m}^{+}} \mu_{i} p_{i} m_{i}^{h} \leqslant p_{1} \operatorname{mult}_{B}\left(Z^{h} \circ F\right)+\left(\Sigma_{s}-p_{1}\right) \operatorname{mult}_{B_{1}}\left(Z^{h} \circ F\right)^{1} .
$$

В частности,

$$
\sum_{i \in J_{s} \cup J_{m}^{+}} \mu_{i} p_{i} m_{i}^{h} \leqslant \Sigma_{s} \operatorname{mult}_{B}\left(Z^{h} \circ F\right) .
$$

2.2. Доказательство предложения 2.1: подсчет кратностей. Рассмотрим сначала следуюшую обшую ситуацию. Пусть $Y \subset V$-неприводимое горизонтальное подмногообразие коразмерности $2, Y^{i} \subset V_{i}$ - его собственньй прообраз,

$$
m_{Y}(i)=\operatorname{mult}_{B_{i-1}} Y^{i-1}
$$

- соответствуюшая кратность. Положим $Y_{F}=(Y \circ F)$ - эффективный цикл коразмерности два в слое $F, Y_{F}^{i} \subset V_{i}$ - его собственньй прообраз,

$$
m_{Y, F}(i)=\operatorname{mult}_{B_{i-1}} Y^{i-1}
$$

Поскольку носитель цикла $Y_{F}$ содержится в слое $F$, числа $m_{Y, F}(i)$ обрашаются в нуль при $i \in J_{m}^{-}$. 
Лемма 2.1. Имеет место оценка

$$
\sum_{i \in J_{s} \cup J_{m}^{+}} p_{i} m_{Y}(i) \mu_{i} \leqslant \sum_{i \in J_{s}} p_{i} m_{Y, F}(i) .
$$

Прежде чем начинать доказательство, напомним некоторые факты, непосредственно вытекающие из элементарной теории пересечений [20]. Отсутствие удобных ссылок объясняется тем, что здесь речь идет о пересечении дивизора и подмногообразия, а не двух дивизоров, как в [5], [8]. Пусть $X$ - произвольное гладкое многообразие, $B \subset X, B \not \subset \operatorname{Sing} X$ - неприводимое подмногообразие коразмерности $\geqslant 2, \sigma_{B}: X(B) \rightarrow X-$ его раздутие, $E(B)=\sigma_{B}^{-1}(B)-$ исключительный дивизор. Пусть

$$
Z=\sum m_{i} Z_{i}, \quad Z_{i} \subset E(B),
$$

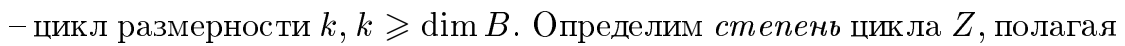

$$
\operatorname{deg} Z=\sum_{i} m_{i} \operatorname{deg}\left(Z_{i} \cap \sigma_{B}^{-1}(b)\right)
$$

где $b \in B$ - точка общего положения, $\sigma_{B}^{-1}(b) \cong \mathbb{P}^{\operatorname{codim} B-1}$ и степень справа есть обычная степень в проективном пространстве.

Отметим, что $\operatorname{deg} Z_{i}=0$ тогда и только тогда, когда $\sigma_{B}\left(Z_{i}\right)-$ собственное замкнутое подмножество подмногообразия $B$.

Теперь пусть $D$ - простой дивизор Вейля на $X, Y \subset X$ - некоторое неприводимое подмногообразие размерности $l \leqslant \operatorname{dim} X-1$. Предположим, что $Y \not \subset D$ и что $\operatorname{dim} B \leqslant l-1$. Собственные прообразы дивизора $D$ и подмногообразия $Y$ на $X(B)$ обозначим символами $D^{B}$ и $Y^{B}$ соответственно.

Лемма 2.2. (i) Предположим, ито $\operatorname{dim} B \leqslant l-2$. Тогда

$$
D^{B} \circ Y^{B}=(D \circ Y)^{B}+Z
$$

где о обозначает операцию взятия алгебраического иикла теоретико-схемного пересечения, $\operatorname{Supp} Z \subset E(B)$ и

$$
\operatorname{mult}_{B}(D \circ Y)=\operatorname{mult}_{B} D \cdot \operatorname{mult}_{B} Y+\operatorname{deg} Z .
$$

(ii) Предположим, что $\operatorname{dim} B=l-1$. Тогда

$$
D^{B} \circ Y^{B}=Z+Z_{1}
$$

где $\operatorname{Supp} Z \subset E(B), \operatorname{Supp} \sigma_{B}\left(Z_{1}\right)$ не содержит $B$ и

$$
D \circ Y=\left[\left(\operatorname{mult}_{B} D\right)\left(\operatorname{mult}_{B} Y\right)+\operatorname{deg} Z\right] B+\left(\sigma_{B}\right)_{*} Z_{1} .
$$

Доказательство легко получается применением стандартной теории пересечений [20]. 
2.3. Доказательство леммы 2.1. Построим последовательность эффективных циклов коразмерности три на многообразиях $V_{i}$, полагая

$$
\begin{aligned}
& Y \circ F=Z_{0}\left(=Y_{F}\right), \\
& Y^{1} \circ F^{1}=Z_{0}^{1}+Z_{1}, \\
& \ldots \ldots \ldots \ldots \ldots \ldots \ldots \ldots \ldots \ldots \ldots \ldots \ldots \ldots \ldots \\
& Y^{i} \circ F^{i}=\left(Y^{i-1} \circ F^{i-1}\right)^{i}+Z_{i}, \\
& \ldots \ldots \ldots \ldots \ldots \ldots \ldots \ldots \ldots \ldots
\end{aligned}
$$

$i \in J_{s}$, где $\operatorname{Supp} Z_{i} \subset E_{i}$. Таким образом, для любого $i \in J_{s}$ получаем:

$$
Y^{i} \circ F^{i}=Y_{F}^{i}+Z_{1}^{i}+\cdots+Z_{i-1}^{i}+Z_{i}
$$

Для любых $j>i, j \in J_{s}$ положим

$$
m_{i, j}=\operatorname{mult}_{B_{j-1}}\left(Z_{i}^{j-1}\right)
$$

(кратность неприводимого подмногообразия вдоль меньшего подмногообразия понимается в обычном смысле; для произвольного цикла продолжаем кратность по линейности).

Положим теперь $d_{i}=\operatorname{deg} Z_{i}$. Получаем следуюшую систему равенств:

$$
\begin{aligned}
& m_{Y}(1) \mu_{1}+d_{1}=m_{Y, F}(1), \\
& m_{Y}(2) \mu_{2}+d_{2}=m_{Y, F}(2)+m_{1,2} \text {, }
\end{aligned}
$$

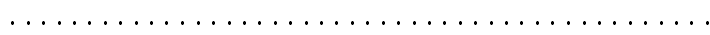

$$
\begin{aligned}
& m_{Y}(i) \mu_{i}+d_{i}=m_{Y, F}(i)+m_{1, i}+\cdots+m_{i-1, i}
\end{aligned}
$$

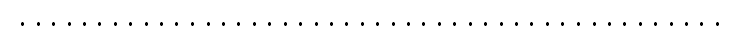

$i \in J_{s}$. Полагая $S=\max \left\{i \in J_{s}\right\}$, рассмотрим последнее равенство в этой последовательности:

$$
m_{Y}(S) \mu_{S}+d_{S}=m_{Y, F}(S)+m_{1, S}+\cdots+m_{S-1, S} .
$$

Если $J_{m}^{+} \neq \varnothing$, то согласно части (ii) леммы 2.2 имеем

$$
d_{S} \geqslant \sum_{i \in J_{m}^{+}} m_{Y}(i) \mu_{i} \operatorname{deg}\left(\varphi_{i-1, S}\right)_{*} B_{i-1} \geqslant \sum_{i \in J_{m}^{+}} m_{Y}(i) \mu_{i} .
$$

Напомним следующее полезное

ОПРЕДЕЛЕНИЕ 2.1 (см. [5], [8]). Функция $a: J_{s} \rightarrow \mathbb{R}_{+}$называется согласованной со структурой графа, если

$$
a(i) \geqslant \sum_{\substack{j \rightarrow i \\ j \in J_{s}}} a(j)
$$

для любого $i \in J_{s}$.

Фактически мы будем использовать только одну функцию, согласованную со структурой графа, а именно $a(i)=p_{i}$. 
ПРЕДЛОЖЕНИЕ 2.2. Пусть $а(\cdot)$ - функция, согласованная со структурой графа. Тогда имеет место неравенство

$$
\sum_{i \in \mathscr{J}_{s}} a(i) m_{Y, F}(i) \geqslant \sum_{i \in \mathscr{J}_{S}} a(i) m_{Y}(i) \mu_{i}+a(S) \sum_{i \in \mathscr{J}_{m}^{+}} m_{Y}(i) \mu_{i}
$$

ДоКАЗАТЕЛЬСТво проводится дословно тем же способом, что и в случае двух дивизоров [5], [8]: умножим $i$-е равенство на $a(i)$ и сложим их все вместе. Справа для любого $i \geqslant 1$ получим выражение

$$
\sum_{j \geqslant i+1} a(j) m_{i, j}
$$

В левой части для любого $i \geqslant 1$ получим слагаемое $a(i) d_{i}$.

Лемма 2.3. Если $m_{i, j}>0$, mo $j \rightarrow i$.

ДокаЗАтЕльство [5], [8]. Если $m_{i, j}>0$, то $B_{j-1} \subset \operatorname{Supp} Z_{i}^{j-1}$, но $\operatorname{Supp} Z_{i} \subset E_{i}$, так что $B_{j-1} \subset E_{i}^{j-1}$. Лемма доказана.

Следуюший стандартньй шаг - сравнение кратностей $m_{i, j}$ со степенями.

ЛЕмма 2.4. Для любых $i<j \in J_{s}$ имеем

$$
m_{i, j} \leqslant d_{i}
$$

ДокАЗАТЕЛьство. Если $m_{i, j}=0$, то доказывать нечего. В противном случае $j \rightarrow i$ и необходимо доказать, что

$$
\operatorname{mult}_{B_{j-1}} Z_{i}^{j-1} \leqslant \operatorname{deg} Z_{i}
$$

Учитывая, что отображения $\varphi_{a, b}: B_{a} \rightarrow B_{b}$ сюръективны, достаточно доказать неравенство

$$
\operatorname{mult}_{\left[B_{j-1} \cap \varphi_{i, i-1}^{-1}(t)^{j-1}\right]}\left[Z_{i} \cap \varphi_{i, i-1}^{-1}(t)\right]^{j-1} \leqslant \operatorname{deg}\left[Z_{i} \cap \varphi_{i, i-1}^{-1}(t)\right]
$$

где $t \in B_{i-1}$ - точка общего положения. Учитьвая, что $\varphi_{i, i-1}^{-1}(t)$ есть проективное пространство $\mathbb{P}^{\text {codim } B_{i-1}-1}$, получаем, что справа в (20) стоит обычная степень гиперповерхности в проективном пространстве, а множество $\left[Z_{i} \cap \varphi_{i, i-1}^{-1}(t)\right]^{j-1}$ получается из этой гиперповерхности конечной последовательностью раздутий $\varphi_{s, s-1}, s=i+1, \ldots, j-1$, ограниченных на $\varphi_{i, i-1}^{-1}(t)$. Учитывая, что кратности при раздутиях не возрастают, сводим утверждение к очевидному случаю гиперповерхности в проективном пространстве. Лемма доказана.

В результате получаем следующую оценку:

$$
\sum_{j \geqslant i+1} a(j) m_{i, j}=\sum_{\substack{j \geqslant i+1 \\ m_{i, j} \neq 0}} a(j) m_{i, j} \leqslant d_{i} \sum_{j \rightarrow i} a(j) \leqslant a(i) d_{i}
$$


В силу сказанного выше можно выбросить справа все слагаемые $m_{i, *}, i \geqslant 1$, а слева - все слагаемые $d_{i}, i \geqslant 1$, заменяя при этом знак равенства на знак неравенства $\leqslant$. Что и требовалось доказать.

Полагая в неравенстве $(19) a(i)=p_{i}$ и замечая, что для $j \geqslant S$ имеем $p_{j} \leqslant p_{S}$, завершаем доказательство леммы 2.1.

Закончим доказательство предложения 2.1 .

Очевидно, неравенство (18) остается справедливым, если $Y$ - эффективный горизонтальный цикл коразмерности 2 на $V$, т.е. каждая неприводимая компонента цикла $Y$ - горизонтальное подмногообразие. При этом формулы $(16),(17)$ продолжаются по линейности на множество всех эффективных горизонтальных циклов, а левая и правая части неравенства (18) линейны по $m_{Y}(\cdot), m_{Y, F}(\cdot)$ соответственно.

Положим теперь $Y=Z^{h}$ и учтем, что

$$
m_{Y, F}(i) \leqslant \operatorname{mult}_{B_{1}}\left(Z^{h} \circ F\right)^{1}
$$

для $i \geqslant 2$. Отсюда получаем неравенство (14). Второе неравенство предложения 2.1 вытекает из (14). Предложение доказано.

ЗАмечАниЕ 2.1. Неравенство (15) компактнее, чем (14), однако в некоторых случаях можно получить более сильную оценку на mult $B_{1}\left(Z^{h} \circ F\right)^{1}$, чем на $\operatorname{mult}_{B}\left(Z^{h} \circ F\right)$.

2.4. Оценка кратностей линейной системы: неособый случай. Обратимся теперь к основной задаче - оценке особенностей подвижной линейной системы $\Sigma$. Предположим, что расслоение $V / \mathbb{P}^{1}$ удовлетворяет условиям (v) и (vs), а также в каждой точке $o \in V$ удовлетворяет хотя бы одному из сформулированных ниже условий (f) и (fs):

(f) для любого неприводимого подмногообразия $Y \ni o \in F$ коразмерности 2 (относительно слоя $F$ ) имеет место неравенство

$$
\frac{\text { mult }_{o}}{\operatorname{deg}} Y \leqslant \frac{4}{\operatorname{deg} V}
$$

(fs) $o \in F-$ двойная точка слоя, для любого неприводимого подмногообразия $Y \subset F$ коразмерности 2 имеет место оценка

$$
\frac{\text { mult }_{o}}{\operatorname{deg}} Y \leqslant \frac{6}{\operatorname{deg} V}
$$

и, кроме того, для любой бесконечно близкой точки первого порядка $x \in$ $E_{F}$, где $\varphi_{o}: \widetilde{F} \rightarrow F-$ раздутие точки $o \in F, E_{F} \subset \widetilde{F}-$ исключительный дивизор, выполнено неравенство

$$
\frac{\operatorname{mult}_{x} \tilde{Y}}{\operatorname{deg} Y} \leqslant \frac{3}{\operatorname{deg} V},
$$

где $\widetilde{Y}$ - собственный прообраз подмногообразия $Y$ на $\widetilde{F}$. 
ЗАмечаниЕ 2.2. Подчеркнем, что в условии (f) не оговаривается особая точка $o \in F$ или гладкая. Для общих многообразий, рассматриваемых в данной работе, все гладкие точки удовлетворяют условию (f), а особые точки ведут себя по-разному. Этот вопрос обсуждается ниже в $\S 3$.

Предположим сначала, что $B=\operatorname{centre}\left(\nu_{E}, V\right) \not \subset \operatorname{Sing} F$. Иными словами, либо слой $F$ неособ, либо $B$ не есть особая точка слоя. В силу условия регулярности для любого неприводимого подмногообразия $Y \subset F$ коразмерности два (относительно $F$ ) имеет место оценка (21) для точки общего положения $o \in B$. Все числа $\mu_{i}=1$. Из неравенства (21) немедленно получаем оценку

$$
\sum_{i \in J_{s} \cup J_{m}^{+}} p_{i} m_{i}^{h} \leqslant 4 n^{2} \Sigma_{s}
$$

Поскольку $m_{i}^{h} \leqslant m_{1}^{h} \leqslant 4 n^{2}$, получаем неравенство

$$
\sum_{i \in J_{l}} p_{i} m_{i}^{h} \leqslant 4 n^{2}\left(\Sigma_{s}+\Sigma_{m}^{-}\right) .
$$

Это и есть необходимая оценка на особенности горизонтальной компоненты $Z^{h}$. Рассмотрим вертикальную компоненту $Z^{v}$. В силу условия (v) имеет место неравенство

$$
m_{i}^{v} \leqslant m_{1}^{v} \leqslant \frac{2}{\operatorname{deg} V} d_{v}
$$

Из обобщенного $K^{2}$-условия глубины $\varepsilon$ получаем оценку

$$
\frac{d_{v}}{\operatorname{deg} V}<\frac{2 e n}{\nu_{E}(F)}+\varepsilon n^{2} .
$$

Комбинируя (24) и (25), получаем неравенство

$$
\sum_{i \in J_{s} \cup J_{m}^{+}} p_{i} m_{i}^{v}<2 n\left(\frac{2 e}{\nu_{E}(F)}+\varepsilon n\right)\left(\Sigma_{s}+\Sigma_{m}^{+}\right) .
$$

Учитьвая, что по определению $\nu_{E}(F)=\sum_{i=1}^{k} p_{i} \mu_{i} \geqslant \Sigma_{s}+\Sigma_{m}^{+}$, получаем окончательно:

$$
\sum_{i \in J_{s} \cup J_{m}^{+}} p_{i} m_{i}^{v}<4 n e+2 \varepsilon n^{2}\left(\Sigma_{s}+\Sigma_{m}^{+}\right) .
$$

Теперь из неравенств (6), (23) и (26) вытекает следующая оценка:

$$
\begin{aligned}
& \left(4 n^{2}\left(\Sigma_{s}+\Sigma_{m}^{-}\right)+4 n e+2 \varepsilon n^{2}\left(\Sigma_{s}+\Sigma_{m}^{+}\right)\right)\left(\Sigma_{s}+\Sigma_{m}+\Sigma_{u}\right) \\
& \quad>\left(\left(3 \Sigma_{s}+2 \Sigma_{m}+\Sigma_{u}\right) n+e\right)^{2} .
\end{aligned}
$$

Полагая в этом неравенстве $\varepsilon=2$ и учитывая, что $\Sigma_{m}=\Sigma_{m}^{+}+\Sigma_{m}^{-}$(это ключевой момент), посленесложных арифметических преобразований получаем неравенство

$$
\left(n\left(\Sigma_{s}-\Sigma_{u}\right)+e\right)^{2}<0 .
$$

Противоречие.

Равенство порогов канонического присоединения

$$
c_{\mathrm{virt}}(\Sigma)=c(\Sigma)
$$

в неособом случае $B \not \subset \operatorname{Sing} F$ доказано. 
2.5. Оценка кратностей линейной системы: особый случай. Рассмотрим теперь случай, когда $B=o \in F$ - особая точка слоя. Если многообразие $F$ удовлетворяет в точке $о$ условию (f), то рассуждения предыдушего пункта проходят без изменений. Если это не так, то $\operatorname{deg} V=\operatorname{deg} F \geqslant 6$ (в противном случае $\operatorname{deg} V \leqslant 4$ и условие (f) выполнено автоматически) и потому $\operatorname{dim} F \geqslant 4$, $\operatorname{dim} V \geqslant 5$. Поэтому дискрепантность первого исключительного дивизора $E_{1}$ не меньше 4 (раздувается гладкая точка $o \in V$ ). По предположению выполнено условие $(\mathrm{fs})$, откуда следует неравенство

$$
m_{i}^{h} \leqslant m_{1}^{h} \leqslant 3 n^{2},
$$

так что из оценки (14) получаем

$$
\sum_{i \in J_{l}} p_{i} m_{i}^{h} \leqslant 3 n^{2}\left(p_{1}+\Sigma_{s}+\Sigma_{m}^{-}\right)
$$

$\mathrm{C}$ другой стороны, как было отмечено вьше, $a\left(E_{1}, V\right) \geqslant 4$, так что в неравенстве (6) можно заменить числитель на $\left(p_{1}+3 \Sigma_{s}+2 \Sigma_{m}+\Sigma_{u}\right)$. Теперь, рассуждая как в п. 2.4, получаем неравенство

$$
\begin{gathered}
\left(n\left(\Sigma_{s}-\Sigma_{u}\right)+e\right)^{2}+\left(\Sigma_{s}-3 p_{1}\right)\left(\Sigma_{s}+\Sigma_{m}+\Sigma_{u}\right) n^{2} \\
\quad+n p_{1}\left(n p_{1}+2\left(3 \Sigma_{s}+2 \Sigma_{m}+\Sigma_{u}\right) n+2 e\right)<0 .
\end{gathered}
$$

Очевидно, $\Sigma_{s}-3 p_{1} \geqslant-2 p_{1}$, так что опять получаем противоречие.

Равенство порогов канонического присоединения $c_{\mathrm{virt}}(\Sigma)=c(\Sigma)$ доказано полностью.

Ввиду важности полученного результата для дальнейшей работы сформулируем его в качестве отдельного утверждения.

ТЕОРема 2. Предположим, что расслоение $V / \mathbb{P}^{1}$ удовлетворяет обобщенному $K^{2}$-условию глубины 2 , условиям (v), (vs) и хотя бъ одному из условий (f) или (fs) в любой точке $о \in V$. Тогда для любой подвижной линейной системы $\Sigma \subset\left|-n K_{V}+l F\right|$ c $l \in \mathbb{Z}_{+}$ее виртуальный и фактический пороги канонического присоединения совпадают:

$$
c_{\mathrm{virt}}(\Sigma)=c(\Sigma)
$$

\section{§3. Многообразия с пучком двойных накрытий Фано}

Для того чтобы применить развитую выше технику к расслоениям $V / \mathbb{P}^{1}$ на двойные гиперповерхности Фано, необходимо описать подвижные линейные системы на этих многообразиях и проверить условия на кратности горизонтальных и вертикальных циклов для рассматриваемых семейств $1-8$ и $1^{*}-5^{*}$ пп. 0.2 и 0.3 .

3.1. Подвижные системы на многообразиях типа $((0),(2,0))$. Пусть $V / \mathbb{P}^{1}$ - многообразие из семейства $((0),(2,0))$. Конструкция многообразия $V$ допускает следующее альтернативное описание.

Пусть $W_{\mathbb{P}} \subset \mathbb{P}$ - гиперповерхность степени $2 l$,

$$
\sigma_{\mathbb{Y}}: \mathbb{Y} \rightarrow \mathbb{P}
$$

- двойное накрытие, разветвленное над дивизором $W_{\mathbb{P}}$. Рассмотрим многообразие $Y=\mathbb{P}^{1} \times \mathbb{Y}$, которое реализуется как двойное накрытие $\sigma_{Y}: Y \rightarrow X=\mathbb{P}^{1} \times \mathbb{P}$, 
разветвленное над дивизором $W=\mathbb{P}^{1} \times W_{\mathbb{P}}$. Положим $V=\sigma_{Y}^{-1}(Q)$, где $Q \subset X$ - гладкий дивизор типа $(2, m)$. Легко видеть, что описанный способ построения многообразия $V$ совпадает с конструкцией п. 0.2 .

По предположению дивизор $Q \subset \mathbb{P}^{1} \times \mathbb{P}$ задается уравнением

$$
A\left(x_{*}\right) u^{2}+2 B\left(x_{*}\right) u v+C\left(x_{*}\right) v^{2}=0,
$$

где $A(\cdot), B(\cdot), C(\cdot)$ однородны степени $m$. Здесь $(u: v)$ и $\left(x_{*}\right)=\left(x_{0}: \ldots: x_{M+1}\right)$ - однородные координаты на $\mathbb{P}^{1}$ и $\mathbb{P}$ соответственно.

Далее, пусть $H_{\mathbb{P}}-$ класс гиперплоскости в $\mathbb{P}, L_{X}=p_{X}^{*} H_{\mathbb{P}}$ - тавтологический класс на $X$, где $p_{X}: X \rightarrow \mathbb{P}$ - проекция на второй сомножитель, $L_{V}=\left.\sigma_{Y}^{*} L_{X}\right|_{V}$. Легко видеть, что

$$
K_{V}=-L_{V}
$$

так что антиканоническая линейная система $\left|-K_{V}\right|$ свободна и задает проекцию $p_{V}=p_{X} \circ \sigma: V \rightarrow \mathbb{P}$.

ЛЕмма 3.1. Проекция $p_{V}$ пропускается через двойное накрвтие $\sigma_{\mathbb{Y}}: \mathbb{Y} \rightarrow \mathbb{P}$. Точнее, существует морфизм $p: V \rightarrow \mathbb{Y}$ такой, что

$$
p_{V}=\sigma_{\mathbb{Y}} \circ p .
$$

Степень морфизма р в общей точке равна 2.

ДокаЗАтЕльство. Рассмотрим точку $x \in \mathbb{P} \backslash W_{\mathbb{P}}$ общего положения, пусть $\left\{y^{+}, y^{-}\right\}=\sigma_{\mathbb{Y}}^{-1}(x) \subset \mathbb{Y}$. Положим

$$
L_{x}=\mathbb{P}^{1} \times\{x\} \subset X, \quad L_{x}^{ \pm}=\mathbb{P}^{1} \times\left\{y^{ \pm}\right\} \subset Y .
$$

Очевидно, прообраз $\sigma_{Y}^{-1}\left(L_{x}\right)$ есть дизъюнктное объединение прямых $L_{x}^{+}$и $L_{x}^{-}$, причем

$$
p_{\mathbb{Y}}\left(L_{x}^{ \pm}\right)=y^{ \pm},
$$

где $p_{Y}: Y \rightarrow \mathbb{Y}$ - проекция на второй сомножитель. Дивизор $Q$ пересекает $L_{x}$ в двух различных (для общей точки $x$ ) точках $q_{1}, q_{2}$. Положим

$$
\sigma^{-1}\left(q_{i}\right)=\left\{o_{i}^{+}, o_{i}^{-}\right\} \subset V, \quad o_{i}^{ \pm} \in L_{x}^{ \pm} .
$$

Морфизм $p$ есть ограничение $\left.p_{Y}\right|_{V}$. Очевидно,

$$
p^{-1}\left(y^{ \pm}\right)=\left\{o_{1}^{ \pm}, o_{2}^{ \pm}\right\}
$$

где знак + или - выбирается один и тот же справа и слева. Этим лемма доказана.

Пусть $\Delta \subset V$-подмногообразие коразмерности 2 , заданное системой уравнений $A=B=C=0$. Подмногообразие $\Delta$ заметается прямыми $L_{y}=\mathbb{P}^{1} \times\{y\}$, которые стягиваются морфизмом $p$. Положим $\Delta_{\mathbb{Y}}=p(\Delta)$. Очевидно,

$$
p: V \backslash \Delta \rightarrow \mathbb{Y} \backslash \Delta_{\mathbb{Y}}
$$

- конечный морфизм степени 2. Пусть $\tau \in \operatorname{Bir} V$ - соответствующая инволюция Галуа. Легко видеть, что $\tau$ коммутирует с инволюцией Галуа $\alpha \in$ Aut $V$ двойного накрытия $\sigma: V \rightarrow Q$, так что $\tau$ и $\alpha$ порождают группу из четырех элементов. Поскольку инволюция $\tau$ бирегулярна вне инвариантного замкнутого множества $\Delta$ коразмерности 2, $\tau \in \operatorname{Aut}(V \backslash \Delta)$, действие $\tau$ на группе Пикара $\operatorname{Pic} V$ корректно определено.

Пусть $\Sigma \subset\left|-n K_{V}+l F\right|-$ подвижная линейная система. 
Лемма 3.2. (i) Инволюция $\tau$ переводит пучок $|F|$ слоев морфизма $\pi$ в пучок $\left|m L_{V}-F\right|$.

(ii) $E$ сли $l<0$, то инволющия $\tau$ переводит линейную систему $\Sigma$ в линейную систему

$$
\Sigma^{+} \subset\left|n^{+} L_{V}+l^{+} F\right|
$$

əде $n^{+}=n+l m \geqslant 0, l^{+}=-l>0$.

ДокаЗАтельство. Очевидно, $\tau^{*} L_{V}=L_{V}$. Пусть $F_{t}=\pi^{-1}(t)-$ некоторый слой. Имеем

$$
p^{-1}\left(p\left(F_{t}\right)\right)=F_{t} \cup \tau\left(F_{t}\right) .
$$

Но $p\left(F_{t}\right) \sim m H_{\mathbb{Y}}=m \sigma_{\mathbb{Y}}^{*} H_{\mathbb{P}}$ в силу конструкции многообразия $V$. Поскольку $p^{*} H_{\mathbb{Y}}=L_{V}$, получаем утверждение (i). Таким образом, $\tau^{*} F=m L_{V}-F$. Отсюда непосредственно вытекает второе утверждение леммы.

ЗАмЕчАниЕ 3.1. Многообразия типа $((0),(2,0))$ аналогичны по своим свойствам расслоениям Фано, рассмотренным в [14].

3.2. Проверка $K$-условия. Докажем, что многообразие $V / \mathbb{P}^{1}$, принадлежащее к любому из семейств $2-8$ или $1^{*}-5^{*}$ пп. $0.2,0.3$, удовлетворяет $K$-условию. Зафиксируем подвижную линейную систему $\Sigma \subset\left|-n K_{V}+l F\right|$. Необходимо доказать, что $l \in \mathbb{Z}_{+}$. Для этого будем использовать рассуждения следующих двух типов:

(a) Предположим, что имеется дивизор $E \subset V$, заметаемый семейством неприводимых горизонтальных кривых $\left(C_{\delta}, \delta \in \Delta\right)$ таким, что

$$
\left(-K_{V} \cdot C_{\delta}\right) \leqslant 0
$$

Тогда в силу подвижности линейной системы $\Sigma$ имеем $l \geqslant 0$, что и требуется.

(b) Предположим, что на $V$ имеется горизонтальный простой дивизор $E$, удовлетворяющий неравенству

$$
\left(-K_{V} \cdot E \cdot L_{V}^{M-1}\right) \leqslant 0 .
$$

Для обшего дивизора $D \in \Sigma$ цикл $(D \circ E)$ эффективен и потому

$$
\left(D \cdot E \cdot L_{V}^{M-1}\right) \geqslant 0,
$$

откуда, учитывая, что $\left(H_{F} \cdot L_{V}^{M-1}\right)>0$, получаем $l \geqslant 0$.

Рассмотрим теперь многообразия типов $2-8$ и $1^{*}-5^{*}$ по порядку.

3.2.1. Многообразия семейства $((0),(1,1))$. Здесь $X=\mathbb{P}^{1} \times \mathbb{P}, Q \subset X$ задано уравнением $A u+B v=0$, где $A, B$ - однородные многочлены степени $m$ на $\mathbb{P}$. Пара уравнений $A=B=0$ определяет на $Q$ дивизор $\Delta=\mathbb{P}^{1} \times \Delta_{\mathbb{P}}$, заметаемый горизонтальными прямыми, $\Delta_{\mathbb{P}} \subset \mathbb{P}$ - подмногообразие $\{A=B=0\}$ коразмерности два. Для любой прямой $L_{x}=\sigma^{-1}\left(\mathbb{P}^{1} \times\{x\}\right), x \in \Delta_{\mathbb{P}}$, имеем

$$
\left(-K_{V} \cdot L_{x}\right)=\left(L_{V} \cdot L_{x}\right)=0 .
$$

Рассуждение (а) показывает, что $K$-условие вьполнено. 
3.2.2. Многообразия типа $((1),(0,1))$. Здесь

$$
\mathscr{E}=\mathscr{O}_{\mathbb{P}^{1}}^{\oplus(M+1)} \oplus \mathscr{O}_{\mathbb{P}^{1}}(1),
$$

и пусть $E \subset X$ - дивизор обших нулей всех сечений $s \in \mathscr{O}_{\mathbb{P} 1}(1)$. Очевидно, $E=$ $\mathbb{P}^{1} \times \mathbb{P}^{M}$. Пусть $L_{E}=p_{E}^{*}\left(\left.H_{\mathbb{P}}\right|_{\mathbb{P}^{M}}\right)$ - тавтологический класс на $E$, где $p_{E}: E \rightarrow \mathbb{P}^{M}$ - проекция на второй сомножитель. Имеем

$$
-\left.K_{V}\right|_{E}=\left.L_{V}\right|_{E}=L_{E},
$$

$E \cap Q \sim m L_{E}$, так что $E \cap Q=\mathbb{P}^{1} \times Q_{E}$, где $Q_{E} \subset \mathbb{P}^{M}$ - гиперповерхность степени $m$. Таким образом, $V$ содержит дивизор $\sigma^{-1}(E \cap Q)$, заметаемый кривыми $L_{x}=\sigma^{-1}(\mathbb{P} \times\{x\}), x \in Q_{E}$, на которых $\left(-K_{V}\right)$ тривиален. Согласно рассуждению (а) $K$-условие выполнено.

3.2.3. Многообразия типа $((2),(1,0))$. Здесь $-K_{V}=L_{V}-F$,

$$
\mathscr{E}=\mathscr{O}_{\mathbb{P}^{1}}^{\oplus(M+1)} \oplus \mathscr{O}_{\mathbb{P}^{1}}(2),
$$

пусть $E \subset X$ - дивизор общих нулей сечений $s \in \mathscr{O}_{\mathbb{P}^{1}}(2)$, тогда

$$
\sigma^{-1}(E \cap Q) \in\left|L_{V}-2 F\right| .
$$

Нетрудно подсчитать, что

$$
\left(-K_{V} \cdot\left(L_{V}-2 F\right) \cdot L_{V}^{M-1}\right)=2 n(1-m) \leqslant 0 .
$$

Согласно рассуждению (b) $K$-условие справедливо.

3.2.4. Многообразия типа $((2),(0,0))$. Здесь $-K_{V}=L_{V}$ и применимо рассуждение (a): дивизор $\sigma^{-1}(E \cap Q) \subset V$ (в обозначениях предыдушего случая) заметается горизонтальными кривыми, на которых класс $L_{V}$ тривиален.

3.2.5. Многообразия типа $((3),(0,0))$. Здесь

$$
\mathscr{E}=\mathscr{O}_{\mathbb{P}^{1}}^{\oplus(M+1)} \oplus \mathscr{O}_{\mathbb{P}^{1}}(3)
$$

и $-K_{V}=L_{V}-F$. Пусть $E \subset X-$ дивизор общих нулей сечений $s \in \mathscr{O}_{\mathbb{P}^{1}}(3)$, тогда дивизор $\sigma^{-1}(E \cap Q) \subset V$ заметается горизонтальными кривьми, на которых $L_{V}$ тривиален. Применяем рассуждение (а).

3.2.6. Многообразия типа $((1,2),(0,0))$. Здесь $-K_{V}=L_{V}-F$ и сушествует простой дивизор $E \subset V$ такой, что

$$
E \sim L_{V}-2 F
$$

Легко проверить, что

$$
\left(\left(L_{V}-2 F\right) \cdot\left(L_{V}-F\right) \cdot L_{V}^{M-1}\right)=0 .
$$

Согласно рассуждению (b) $K$-условие выполнено. 
3.2.7. Многообразия типа $((1,1,1),(0,0))$. Здесь $-K_{V}=L_{V}-F$, причем

$$
\operatorname{codim}_{V} \operatorname{Bs}\left|L_{V}-F\right|=3 .
$$

Поэтому для любого псевдоэффективного класса $D \in \operatorname{Pic} V$ имеем неравенство

$$
\left(D \cdot\left(L_{V}-F\right)^{2} \cdot L_{V}^{M-2}\right) \geqslant 0 .
$$

Однако нетрудно подсчитать, что $\left(\left(L_{V}-F\right)^{3} \cdot L_{V}^{M-2}\right)=0$. Поэтому если $D=$ $-n K_{V}+l F$ есть даже просто псевдоэффективный класс, то $l \in \mathbb{Z}_{+}$. В частности, $K$-условие выполнено.

Оставшиеся пять типов двойных пространств рассматриваются аналогично (с упрошениями).

Справедливость $K$-условия для многообразий $2-8$ и $1^{*}-5^{*}$ доказана.

3.3. Доказательство бирациональной жесткости. Теперь для того чтобы доказать теорему 1 , необходимо установить справедливость равенства

$$
c_{\mathrm{virt}}(\Sigma)=c(\Sigma)
$$

для любой подвижной линейной системы $\Sigma \subset\left|-n K_{V}+l F\right|$ с $n \geqslant 1$ и $l \in \mathbb{Z}_{+}$. В свою очередь, в силу теоремы 2 достаточно проверить, что для многообразий типов $1-8$ и $1^{*}-5^{*}$ справедливо обобщенное $K^{2}$-условие глубины 2 и что регулярное расслоение $V / \mathbb{P}^{1}$ удовлетворяет условиям (v), (vs) и (f) или (fs) в каждой точке (условие (h) непосредственно вытекает из любого из двух условий (f) или $(\mathrm{fs})$ ). Условия (v) и (vs) проверены в [1], условие (f) для регулярной гладкой точки слоя $o \in F$ - в работе [4]. Осталось проверить, что в двойной точке $o$ слой $F$ удовлетворяет хотя бы одному из двух условий (f) или (fs).

Если особая точка $o \in F$ лежит вне дивизора ветвления морфизма $\sigma$, то удовлетворяется требование (f). Это легко установить стандартным методом гиперкасательных дивизоров. Необходимо учесть, что если $o \in F$ не лежит на дивизоре ветвления, то имеются $l$ дополнительных гиперкасательных дивизоров, связанных с двойным накрытием. Это и позволяет получить оценку (21) для любого неприводимого подмногообразия $Y \subset F$ коразмерности 2 (относительно слоя $F$ ). Мы не приводим здесь этих рассуждений, потому что они стандартны и, в частности, параллельны доказываемой ниже более сильной оценке (с заменой 4 на 3 ) для гладких точек.

Будем считать, что особая точка $o \in F$ лежит на дивизоре ветвления. Пусть $Y \subset F$ - неприводимое подмногообразие коразмерности $2, T=\sigma^{-1}\left(T_{p} G \cap G\right)$, $p=\sigma(o),-$ касательный дивизор. Если $Y \not \subset T$, то, рассматривая эффективньй цикл $(Y \circ T)$ коразмерности 3 и применяя к нему стандартную технику гиперкасательных дивизоров (пересекая его с $D_{i} \in \Lambda_{i}, i=4, \ldots, m-1$ ), получаем справедливость оценки (21). Значит, можно предполагать, что $Y \subset T$. Применяя технику гиперкасательных дивизоров (с $D_{i} \in \Lambda_{i}$ для $\left.i=3, \ldots, m-1\right)$, получаем (22)первое из двух неравенств условия (fs). Докажем второе.

Пусть $\varphi: \widetilde{T} \rightarrow T$ - раздутие точки $o, E=\varphi^{-1}(o) \subset \widetilde{T}$ - исключительный дивизор. Легко видеть, что двойное накрытие $\sigma$ реализует $E$ как двойное накрытие квадрики $E_{G}$ с ветвлением в дивизоре $\widetilde{W} \cap E_{G}-$ сечении $E_{G}$ квадратичной гиперповерхностью. Квадрика $E_{G}$ есть исключительный дивизор раздутия точки 
$p=\sigma(o)$ на многообразии $T_{p} G \cap G, \widetilde{W}-$ собственный прообраз ограничения $\left.W\right|_{G}$. По предположению $Y$ - простой дивизор на $T$. Следовательно, $Y_{E}=(\widetilde{Y} \circ E)-$ эффективный дивизор на двойной квадрике $E$, причем $\operatorname{deg} Y_{E}=\operatorname{mult}_{o} Y$. Пусть $x \in E$ - произвольная точка.

Положим $q=\sigma(x) \in E_{G}, R=\sigma^{-1}\left(T_{q} E_{G} \cap E_{G}\right)$. Дивизор $R \subset E$ неприводим, $\operatorname{deg} R=4$, $\operatorname{lult}_{x} R=2$. Если $Z \subset E$ - простой дивизор, отличный от $R$, то корректно определен цикл $(Z \circ R)$ коразмерности 2 на $E$, причем

$$
\operatorname{deg} Z=\operatorname{deg}(Z \circ R) \geqslant \operatorname{mult}_{x}(Z \circ R) \geqslant 2 \operatorname{mult}_{x} Z .
$$

Отсюда получаем, что

$$
\operatorname{mult}_{x} \tilde{Y} \leqslant \operatorname{mult}_{x} Y_{E} \leqslant \frac{1}{2} \operatorname{deg} Y_{E}=\frac{1}{2} \operatorname{mult}_{o} Y .
$$

Это доказывает второе неравенство условия (fs). Что же касается обобщенного $K^{2}$-условия глубины 2 , то оно вытекает из неравенства

$$
\left(\left(K_{V}^{2}-2 H_{F}\right) \cdot L_{V}^{M-1}\right) \leqslant 0
$$

которое легко проверяется для многообразий типов $1-8$ и $1^{*}-5^{*}$ пп. 0.2 и 0.3 .

Доказательство теоремы 1 этим завершено.

3.4. Кратности подмногообразий коразмерности 2. Оставшаяся часть параграфа посвящена доказательству следуюшего утверждения.

ПРЕДЛОЖЕНИЕ 3.1. Пусть о $\in F-$ неособая точка слоя, лежащая вне дивизора ветвления морфизма $\sigma$. Тогда для любого неприводимого подмногообразия $Y \subset F$ коразмерности два (и, тем самым, для любого әффективного чикла чистой коразмерности два) справедлива оценка

$$
\frac{\text { mult }_{o}}{\operatorname{deg}} Y \leqslant \frac{3}{\operatorname{deg} V}=\frac{3}{2 m} .
$$

ЗАмЕчАниЕ 3.2. Предложение 3.1 намечает другой подход к доказательству бирациональной жесткости рассматриваемых многообразий, основанньй на предложении 1.7. Если неособая точка $o \in F$ лежит на дивизоре ветвления морфизма $\sigma$, то сушествует неприводимое подмногообразие $Y^{+} \subset F$ коразмерности 2 , для которого оценка условия (f) неулучшаема: линейная система $|H-2 o|$ в этом случае подвижна и натянута на два дивизора, $\sigma^{*}\left(T_{p} G \cap G\right)$ и $\sigma^{*}\left(T_{p} W \cap G\right)$, где $p=\sigma(o)$. Пересечение этих дивизоров и дает подмногообразие $Y^{+}$. Можно показать, однако, что для любого другого подмногообразия коразмерности два $Y \neq Y^{+}$оценка (27) справедлива. Что же касается подмногообразия $Y^{+}$, то оно удовлетворяет оценке (27) во всех остальных точках, включая бесконечно близкие точки первого порядка, лежашие над точкой $o$. Теперь по соображениям дискрепантности можно доказывать бирациональную жесткость, основываясь исключительно на предложении 1.7. Однако комбинация этих идей с теоремой 2 приводит к дальнейшему усилению техники, что будет использовано в последующих работах. 
ДоКАЗАТЕЛЬСТво ПРЕДЛОЖЕНИЯ 3.1. Рассуждая по образцу работы [1; § 2], приведем два способа получения оценки (27). Первый гораздо проще, однако проходит для достаточно больших размерностей $M \geqslant M_{0}$. Второй способ дает оценку (27) для всех размерностей, однако требует дополнительных условий общности положения, которые необходимо обосновывать (п. 3.5). При любом способе рассуждений первый шаг дает

ЛЕмма 3.3. Если выполнено неравенство

$$
\frac{\operatorname{mult}_{o}}{\operatorname{deg}} Y>\frac{3}{2 m}
$$

mо $\sigma(Y) \subset T_{p} G$, әде $p=\sigma(o)$.

ДокАЗАТЕЛЬСТво. Предположим противное: $\sigma(Y) \not \subset T_{p} G$. Тогда пересечение $Y \cap T$, где $T=\sigma^{*}\left(T_{p} G \cap G\right)$ - касательное сечение слоя $F$ в точке $o$, имеет коразмерность 3 и потому корректно определен эффективный цикл $(Y \circ T)$. Для некоторой компоненты $Y_{3}$ этого цикла вьполнено неравенство

$$
\frac{\operatorname{mult}_{o}}{\operatorname{deg}} Y_{3}>\frac{3}{m}
$$

Теперь, рассуждая обычным образом, рассмотрим общие дивизоры гиперкасательных линейных систем

$$
D_{i} \in \Lambda_{i}, \quad D_{j}^{+} \in \Lambda_{j}
$$

где

$$
i \in\{4, \ldots, m-1\}, \quad j \in\{l, \ldots, 2 l-2\}
$$

при $m \leqslant 2 l$ и

$$
i \in\{4, \ldots, m-2\}, \quad j \in\{l, \ldots, 2 l-1\}
$$

при $m \geqslant 2 l+1$. Из соображений коразмерности базисного множества системы $\Lambda_{i}$ получаем, что множество

$$
Y_{3} \cap\left(\bigcap_{i} D_{i}\right) \cap\left(\bigcap_{j} D_{j}^{+}\right)
$$

одномерно в окрестностях точки $о$. Отсюда стандартным образом получаем оценку

$$
\frac{\text { mult }_{o}}{\operatorname{deg}} Y_{3} \leqslant \frac{4 l}{m(2 l-1)}
$$

при $m \leqslant 2 l$ и

$$
\frac{\text { mult }_{o}}{\operatorname{deg}} Y_{3} \leqslant \frac{2}{m-1}
$$

при $m \geqslant 2 l+1$. В любом случае оценки (30) и (31) противоречат неравенству (29). Лемма доказана.

Обратимся к доказательству предложения 3.1. Если оценка (27) неверна, т.е. справедлива оценка (28), то согласно доказанному выше $\sigma(Y) \subset T_{p} G$, т.е.

$$
Y \subset T_{1}=\sigma^{-1}\left(G \cap T_{p} G\right) .
$$


В силу условий регулярности $T_{1} \subset F$ - неприводимое подмногообразие с изолированной двойной точкой $o \in T_{1}$. Рассмотрим замкнутое множество

$$
T_{12}=\sigma^{-1}\left(\sigma\left(T_{1}\right) \cap T_{p} \sigma\left(T_{1}\right)\right) .
$$

В силу условий регулярности $T_{12} \subset F$ - неприводимое подмногообразие коразмерности 2 , причем mult $T_{12}=6$, так что справедливо равенство

$$
\frac{\text { mult }_{o}}{\operatorname{deg}} T_{12}=\frac{3}{2 m}
$$

откуда в силу неравенства (28) следует, что $Y \neq T_{12}$. Пусть, как выше,

$$
D_{i} \in \Lambda_{i}, \quad D_{j}^{+} \in \Lambda_{j}^{+}
$$

- общие гиперкасательные дивизоры, где

$$
i \in I=\{2,4, \ldots, m-1\}, \quad j \in J=\{l, \ldots, 2 l-2\}
$$

(в $I$ элемент $i=3$ пропушен) при $m \leqslant 2 l$ и

$$
i \in I\{2,4, \ldots, m-2\}, \quad j \in J=\{l, \ldots, 2 l-1\}
$$

(в $I$ снова пропушено $i=3$ ) при $m \geqslant 2 l+1$. В силу условий регулярности пересечение

$$
Y \cap\left(\bigcap_{i \in I} D_{i}\right) \cap\left(\bigcap_{j \in J} D_{j}^{+}\right)
$$

одномерно в окрестности точки $о$, откуда получаем оценки

И

$$
\frac{\operatorname{mult}_{o}}{\operatorname{deg}} Y \leqslant \frac{8 l}{3 m(2 l-1)}
$$

$$
\frac{\text { mult }_{o}}{\operatorname{deg}} Y \leqslant \frac{4}{3(m-1)}
$$

при $m \leqslant 2 l$ и $m \geqslant 2 l+1$ соответственно. Нетрудно проверить, что получающиеся оценки доказывают предложение 3.1 во всех случаях, кроме следуюших:

$$
m \in\{3,4,5,6,7,8\}, \quad l \in\{3,4\} .
$$

Приведем теперь более тонкий способ рассуждений, требующий сильных условий регулярности, однако работающий во всех размерностях, включая небольшие. Этот способ полностью аналогичен методу доказательства условия (vs) для двойной точки слоя $o \in F$ вне дивизора ветвления в [1]. Поэтому здесь лишь кратко опишем основные шаги доказательства, уделяя основное внимание изменениям, которые необходимо внести в рассуждения работы [1].

Итак, предположим, что для неприводимого многообразия $Y \subset F$ справедливо неравенство (28), противоречащее требуемой оценке (27). Согласно лемме 3.3 $Y \subset T_{1}$. Многообразие $T_{1}$ имеет точку $о$ изолированной факториальной особенностью и $\operatorname{Pic} T_{1}=\mathbb{Z} H_{T}$, где

$$
H_{T}=\left.H_{F}\right|_{T_{1}}
$$

- класс гиперплоского сечения. Пусть $\varphi_{T}: \widetilde{T} \rightarrow T_{1}-$ раздутие точки $o, E_{T} \subset \widetilde{T}-$ (неприводимый) исключительный дивизор, $\widetilde{Y} \subset \widetilde{T}$ - собственный прообраз дивизора $Y$. Имеем

$$
\tilde{Y} \sim \alpha H_{T}-\beta E_{T},
$$

причем $\beta / \alpha>3 / 2$ в силу оценки (28). 
Лемма 3.4. Простой дивизор $T_{12} \subset T_{1}$ заметается семейством кривых $\left\{C_{\delta}, \delta \in \Delta\right\}$, общая кривая которого неприводима и удовлетворяет неравенству

$$
\frac{\text { mult }_{o}}{\operatorname{deg}} C_{\delta}>\frac{2}{3} .
$$

Считая лемму доказанной, рассмотрим собственный прообраз $\widetilde{C}_{\delta} \subset \widetilde{T}$ общей кривой $C_{\delta}, \delta \in \Delta$. Очевидно,

$$
\left(\widetilde{Y} \cdot \widetilde{C}_{\delta}\right)<0
$$

так что $C_{\delta} \subset Y$ и потому $T_{12} \subset Y$. Следовательно, $Y=T_{12}$, что невозможно. Этим предложение 3.1 доказано.

Лемма 3.4 доказывается рассуждениями, полностью аналогичными тем, которые использовались при доказательстве леммы 2.2 работы [1], и мы не будем их повторять, лишш напомним основные шаги. При $m \geqslant 4, l \geqslant 3$ определим последовательность чисел

$$
c_{e}=\#[4, e] \cap \mathscr{M}+\#[3, e] \cap \mathscr{L}, \quad e \in \mathbb{Z}_{+},
$$

где $\mathscr{M}=\{2, \ldots, m-1\}, \mathscr{L}=\{l, \ldots, 2 l-1\}$, и построим упорядочивающую функиию

$$
\chi:\{1, \ldots, m+l-4\} \rightarrow \mathbb{Z}_{+},
$$

полагая $\chi\left(\left[c_{e-1}+1, c_{e}\right] \cap \mathbb{Z}_{+}\right)=e$. В силу условия регулярности для общего набора

$$
\mathbb{D}=\left\{D_{i} \in \Lambda_{\chi(i)}^{P}, i=1, \ldots, m+l-4\right\} \in \Lambda^{P}
$$

гиперкасательных дивизоров замкнутое алгебраическое множество

$$
R_{i}(\mathbb{D})=\bigcap_{j=1}^{i} D_{j} \cap T_{P}
$$

имеет коразмерность $i$ в $T_{12}$ при $i=1, \ldots, m+l-4$. Получаем эффективный 1-цикл

$$
R(\mathbb{D})=R(\mathbb{D})=\left(T_{P} \circ D_{1} \circ \cdots \circ D_{m+l-4}\right)=\sum_{\delta_{i} \in \Delta} C_{\delta_{i}}+\Phi,
$$

где $\left(C_{\delta}, \delta \in \Delta\right)$ - подвижное семейство кривых, заметающее $T_{12}, \Phi$ - неподвижная часть семейства $R(\mathbb{D})$. Легко видеть, что $\Phi$ есть в точности 1 -цикл прямых на $F$, проходящих через точку $o \in F$, т.е. $\operatorname{deg} \Phi=\operatorname{deg} \mathbb{L}(o)$. Следовательно,

$$
\frac{\text { mult }_{o}}{\operatorname{deg}} C_{\delta} \geqslant \frac{\frac{m !}{4} \cdot \frac{(2 l) !}{l !}-\lambda_{m, l}}{\frac{2 m !}{3} \cdot \frac{(2 l-1) !}{(l-1) !}-\lambda_{m, l}}>\frac{2}{3}
$$

в силу предложения 3.2 (доказываемого ниже). Аналогичным образом рассуждаем при $m=3$ или $l=2$. Этим доказательство леммы 3.3 завершено.

Подчеркнем, что в отличие от ситуации, рассмотренной в [1], здесь основная трудность заключается в оценке числа прямых (с учетом кратностей), проходящих через точку $o \in F$. В [1] рассматриваются двойные точки слоев, которых конечное число, так что для общего расслоения $V / \mathbb{P}^{1}$ все прямые, проходящие через особую 
точку слоя, имеют кратность единица, и задача становится тривиальной. В нашем случае $o \in F-$ произвольная точка слоя. Как мы сейчас покажем, кратность прямой, проходящей через некоторую специально выбранную точку $о \in F$, может быть очень велика. Однако не настолько велика, чтобы метод доказательства леммы 2.2 статьи [1] перестал работать в нашем случае. Все остальные различия между доказательством предложения 3.1 настоящей работы и предложения 2.3 в [1] несушественны.

3.5. Оценка числа прямых. Для точки $o \in V \backslash \sigma^{-1}(W)$ вне дивизора ветвления определим алгебраический иикл прямы $x \mathbb{L}(o)$ на $V$, проходящих через точку о, как 0-цикл подсхемы

$$
\left\{q_{1}=\cdots=q_{m}=g_{l+1}=\cdots=g_{2 l}=0\right\}
$$

на $\mathbb{E}=\mathbb{P}\left(T_{p} \mathbb{P}\right) \cong \mathbb{P}^{M}$.

ПРЕДЛОЖЕНИЕ 3.2. Для общего по Зарискому расслоения $V / \mathbb{P}^{1}$ для любой гладкой точки $о \in V \backslash \sigma^{-1}(W)$ имеем

$$
\operatorname{deg} \mathbb{L}(o) \leqslant \lambda_{m, l}=\frac{m !}{6} \frac{(2 l-1) !}{(l-1) !}-1 .
$$

ДокАЗАТЕЛЬСтво. Опишем схему рассуждений и приведем со всеми подробностями основную техническую лемму, позволяющую оценить кратности прямых, проходящих через произвольную точку $o \in V$. Сами вычисления ввиду их элементарности и громоздкости приводить не будем.

Положим

$$
\mathscr{H}=\prod_{i=1}^{m} \mathbb{P}\left(H^{0}\left(\mathbb{P}^{m}, \mathscr{O}_{\mathbb{P}^{M}}(i)\right)\right) \times \prod_{j=l+1}^{2 l} \mathbb{P}\left(H^{0}\left(\mathbb{P}^{M}, \mathscr{O}_{\mathbb{P}^{M}}(j)\right)\right)
$$

Для упрощения обозначений записываем набор ненулевых многочленов $\left(q_{\#}, g_{\#}\right) \in$ $\mathscr{H}$ как

$$
h_{\#}=\left(h_{1}, \ldots, h_{M+1}\right) \in \mathscr{H},
$$

причем, меняя местами прямые сомножители, предполагаем, что $\operatorname{deg} h_{i+1} \geqslant \operatorname{deg} h_{i}$. Пусть

$$
\mathscr{H}^{+} \subset \mathscr{H}
$$

- пространство таких наборов $h_{\#}$, что схема общих нулей многочленов $h_{i}$ нульмерна или пуста. Для $h_{\#} \in \mathscr{H}^{+}{ }_{\text {пусть }} \mathbb{L}\left(h_{\#}\right)-0$-цикл общих нулей системы $h_{\#} \cdot$ Имеет место

ПРЕДЛОЖЕНИЕ 3.3. Коразмерность замкнутого подмножества

$$
\mathscr{H}^{+}(j)=\left\{h_{\#} \in \mathscr{H}^{+} \mid \operatorname{deg} \mathbb{L}\left(h_{\#}\right) \geqslant j\right\}
$$

при $j=\lambda_{m, l}+1$ не меньше чем $M+2$.

Предложение 3.2 вытекает из этого факта автоматически. Объясним, как доказывается предложение 3.3. На самом деле для $j=\lambda_{m, l}+1$ получается гораздо более сильная оценка коразмерности $\operatorname{codim} \mathscr{H}^{+}(j)$, чем $M+2$, но нам это не нужно. 
Прежде всего отметим очевидный факт: при $j=0,1, \ldots, M+2$ имеет место оценка

$$
\operatorname{codim}\left\{h_{\#} \in \mathscr{H}^{+} \mid \# \operatorname{Supp} \mathbb{L}\left(h_{\#}\right) \geqslant j\right\}=j .
$$

Отсюда следует, что можно предполагать, что носитель цикла $\mathbb{L}\left(h_{\#}\right)$ состоит из $j \leqslant M+1$ различных точек (т.е. через любую точку $o \in V$ проходит не более чем $M+1$ различных прямых в слое $F \ni o$ ). Однако кратность этих точек может быть с ростом размерности $M$ очень велика, как показывает следующий

Пример. Пусть $p \in S=\left\{h_{1}=\cdots=h_{M-a}=0\right\}$ - неособая точка, так что $S \ni p$ - росток неособого $a$-мерного подмногообразия. Требование

$$
\operatorname{mult}_{p}\left\{\left.h_{j}\right|_{S}=0\right\} \geqslant 2,
$$

$j=M-a+1, \ldots, M+1$, накладывает на $h_{j}$ ровно $a+1$ условие. С учетом произвольности точки $p$ получаем

$$
a(a+1) \sim a^{2}
$$

условий на многочлены $h_{j}, M-a+1 \leqslant j \leqslant M+1$, и эти условия независимы. При $a \sim \sqrt{M}$, таким образом, на $V$ существуют точки, удовлетворяющие требованию (32). Для таких точек имеем

$$
\operatorname{deg} \mathbb{L}(o) \geqslant 2^{a} \sim 2^{\sqrt{M}} .
$$

Приведенный пример является модельным и показывает порядок роста функции

$$
\max _{o \in V} \operatorname{deg} \mathbb{L}(o)
$$

для общего многообразия $V / \mathbb{P}^{1}$ при $M \rightarrow \infty$. Отметим, однако, что для малых $M$ получаем ту же самую оценку, что и для числа различных прямых

$$
\max _{o \in V} \# \operatorname{Supp} \mathbb{L}(o) .
$$

Рассмотрим теперь задачу оценки коразмерности замкнутого множества $\mathscr{H}^{+}(j)$. Согласно сказанному вьше можно уменьшить множество $\mathscr{H}^{+}$и считать, что

$$
\text { \# Supp } \mathbb{L}\left(h_{\#}\right) \leqslant M+1
$$

для любого $h_{\#} \in \mathscr{H}^{+}$. Для каждого $i=1, \ldots, M$ положим

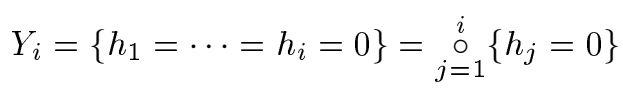

- алгебраический цикл теоретико-схемного пересечения гиперповерхностей $\left\{h_{j}=0\right\}, \operatorname{codim}_{\mathbb{E}} Y_{i}=i$. Положим также

$$
Y_{M+1}=\left\{h_{1}=\cdots=h_{M-1}=h_{M+1}=0\right\} .
$$


Зафиксируем точки $o_{1}, \ldots, o_{k} \in \mathbb{E}, k \leqslant M+1$, и для каждой целочисленной матрицы

рассмотрим множество

$$
\mathbb{M}=\left\|\mu_{i j}\right\|_{\substack{1 \leqslant i \leqslant k \\ 1 \leqslant j \leqslant M+1}} \in \operatorname{Mat}_{k \times(M+1)}\left(\mathbb{Z}_{+}\right)
$$

$$
\mathscr{H}(\mathbb{M})=\left\{h_{\#} \mid \operatorname{mult}_{o_{i}} Y_{j}=\mu_{i j}\right\} \subset \mathscr{H}^{+} .
$$

(Естественно, достаточно рассматривать только матрицы, удовлетворяюшие условию неубывания

$$
\mu_{i, 1} \leqslant \mu_{i, 2} \leqslant \cdots \leqslant \mu_{i, M-1} \leqslant \min \left(\mu_{i, M}, \mu_{i, M+1}\right)
$$

в противном случае либо $\mathscr{H}(\mathbb{M})$ пусто, либо точку $o_{i}$ можно удалить.) Очевидно,

$$
\operatorname{deg} \mathbb{L}\left(h_{\#}\right) \leqslant \sum_{i=1}^{k} \min \left(\mu_{i, M}, \mu_{i, M+1}\right) .
$$

Теперь для доказательства предложения 3.3 необходимо оценить коразмерность

$$
\operatorname{codim}_{\mathscr{H}} \overline{\mathscr{H}(\mathbb{M})}
$$

для всех матриц $\mathbb{M}$, удовлетворяюших оценке

$$
\sum_{i=1}^{k} \min \left(\mu_{i, M}, \mu_{i, M+1}\right) \geqslant \lambda_{m, l}+1 .
$$

Способ оценки коразмерности $\operatorname{codim} \overline{\mathscr{H}(M)}$ при переходе от $Y_{i}$ к $Y_{i+1}$ приведен ниже в п. 3.6. Теперь доказательство предложения 3.3 завершается громоздкими, но совершенно элементарными вычислениями на основе леммы 3.5 , доказываемой ниже. Приводить эти вычисления здесь не будем.

Предложения 3.3 и 3.2 доказаны.

3.6. Метод оценки степени. Пусть $Y \subset \mathbb{A}=\mathbb{C}^{N}$ - эффективный цикл размерности $a \geqslant 1, o_{1}, \ldots, o_{k} \in Y$ - набор попарно различных точек. Положим

$$
\mu_{i}=\operatorname{mult}_{o_{i}} Y,
$$

$i=1, \ldots, k$. Пусть $\mathscr{P}_{e}\left(z_{1}, \ldots, z_{N}\right)$ - пространство всех (неоднородных) многочленов степени, не превосходящей $e \in \mathbb{Z}_{+}$, от переменных $z_{1}, \ldots, z_{N}$. Пусть $c_{1}, \ldots, c_{k} \in$ $\mathbb{Z}_{+}$- такой набор положительных целых чисел, что

$$
c_{1}+\cdots+c_{k-1}+(k-1) \leqslant e, \quad(k-1) a \leqslant N
$$

(если $k=1$, то никаких ограничений не налагается). Положим

$$
\begin{gathered}
a_{1}=\cdots=a_{k-1}=a, \quad a_{k}=\min (a, N-(k-1) a), \\
c_{i}^{*}=c_{i} \text { при } i=1, \ldots, k-1, \\
c_{k}^{*}=\min \left(c_{k}, e-c_{1}-\cdots-c_{k-1}-(k-1)\right) .
\end{gathered}
$$

Пусть

$$
U_{Y}=\left\{f \in \mathscr{P}_{e}\left(z_{*}\right) \mid \operatorname{dim}\{f=0\} \cap \operatorname{Supp} Y=a-1\right\}
$$

- (открытое) множество многочленов, не обрашающихся тождественно в нуль ни на одной компоненте цикла $Y$. Положим

$$
U_{c_{*}}=U\left(c_{1}, \ldots, c_{k}\right)=\left\{f \in U_{Y} \mid \operatorname{mult}_{o_{i}}\left\{\left.f\right|_{Y}=0\right\} \geqslant c_{i} \mu_{i}+1\right\} .
$$

Очевидно, подмножество $U_{c_{*}} \subset U_{Y}$ замкнуто по Зарискому. 
ЛЕмма 3.5. Имеет место оценка

$$
\operatorname{codim} U_{c_{*}} \geqslant \Delta\left(c_{1}, \ldots, c_{k}\right)=\sum_{i=1}^{k}\left(\begin{array}{c}
a_{i}+c_{i}^{*} \\
c_{i}^{*}
\end{array}\right)
$$

ДокаЗАтельство. Пусть $\bar{U}_{c_{*}} \subset \mathscr{P}_{e}\left(z_{*}\right)$ - замыкание множества $U_{c_{*}}$. Для того чтобы доказать неравенство (33), достаточно предъявить замкнутое подмножество $Z \subset \mathscr{P}_{e}\left(z_{*}\right)$, удовлетворяющее равенству

$$
\operatorname{dim} Z=\Delta\left(c_{1}, \ldots, c_{k}\right),
$$

такое, что

$$
Z \cap \bar{U}_{c_{*}}=\{0\} .
$$

Очевидно, $0 \in \bar{U}_{c_{*}}$, так как $\bar{U}_{c_{*}}-$ конус с вершиной в нуле. Способ построения такого замкнутого множества объясним сначала на примере одной точки $o=o_{1}, k=1$, которую без ушерба для обшности считаем началом координат $o=(0, \ldots, 0) \in \mathbb{A}$. Для упрошения обозначений пишем

$$
c_{1}=c_{1}^{*}=c \leqslant e, \quad a_{1}=a \leqslant N, \quad \mu_{1}=\mu .
$$

Пусть $\varphi: \widetilde{\mathbb{A}} \rightarrow \mathbb{A}$ - раздутие точки $o, E=\varphi^{-1}(o) \subset \widetilde{\mathbb{A}}-$ исключительный дивизор $E \cong \mathbb{P}^{N-1}$. Рассмотрим некоторый эффективный дивизор $D \subset \mathbb{A}, o \in D$, не содержаший целиком ни одной компоненты цикла $Y$, так что

$$
\operatorname{dim}(\operatorname{Supp} Y \cap \operatorname{Supp} D)=a-1
$$

и корректно определен эффективный цикл

$$
Y_{D}=(Y \circ D)
$$

размерности $a-1$. Напомним, как вычисляется кратность этого цикла в точке $o$. Пусть $\widetilde{Y}, \widetilde{D} \subset \widetilde{\mathbb{A}}-$ собственные прообразы цикла $Y$ и дивизора $D$ соответственно. Положим

$$
Y_{E}=(\tilde{Y} \circ E)=\sum_{i \in I} b_{i} B_{i}
$$

- проективизированный касательный конус к $Y$ в точке $o \in \operatorname{Supp} Y$. Имеем

$$
(\widetilde{D} \circ \tilde{Y})=(\widetilde{D \circ Y})+\sum_{i \in I} d_{i} B_{i}
$$

для некоторых неотрицательных $d_{i} \in \mathbb{Z}_{+}$. Теперь

$$
\text { mult }_{o} Y_{D}=\mu \text { mult }_{o} D+\sum_{i \in I} d_{i} \operatorname{deg} B_{i}
$$

(это стандартный факт теории пересечений, см. [20]). Отсюда следует, что если $B_{i} \not \subset \widetilde{D}$ для всех $i \in I$, то $d_{i}=0$ и потому имеет место равенство

$$
\operatorname{mult}_{o} Y_{D}=\mu \text { mult }_{o} D \text {. }
$$


Скажем, что набор линейных функций $z_{1}, \ldots, z_{a}$ правильный относительно пары $(o \in Y)$, если

- $B_{i} \not \subset \widetilde{\left(z_{1}\right)}$ для всех $i \in I$;

- проекция $\pi_{\left(z_{1}, \ldots, z_{a}\right)}: \mathbb{C}^{N} \rightarrow \mathbb{C}^{a}$ доминантна на каждой неприводимой компоненте цикла $Y$;

- выполнено следуюшее инфинитезимальное условие в точке $o$. Считая $\left(z_{1}\right.$ : $\left.\ldots: z_{N}\right)$ однородными координатами на $E$, рассмотрим аффинное множество $\left\{z_{1} \neq 0\right\}$ с координатами $\left(y_{2}, \ldots, y_{N}\right)$, где $y_{i}=z_{i} / z_{1}$. Набор функций $\left(y_{2}, \ldots, y_{a}\right)$ определяет проекцию $\iota: \mathbb{C}^{N-1} \rightarrow \mathbb{C}^{a-1}$. Теперь наше условие формулируется таким образом: ограничение

$$
\left.\iota\right|_{B_{i}}: B_{i} \cap\left\{z_{1} \neq o\right\} \rightarrow \mathbb{C}^{a-1}
$$

проекции $\iota$ на каждую компоненту $B_{i}$ есть доминантное отображение.

Легко видеть, что общий набор $\left(z_{1}, \ldots, z_{a}\right)$ линейных функций будет правильньм относительно пары $(o \in Y)$. В силу определения правильности для любого ненулевого многочлена $f \in \mathscr{P}_{e}\left(z_{i}, \ldots, z_{a}\right)$ от переменных $z_{1}, \ldots, z_{a}$ имеем

$$
B_{i} \not \subset \widetilde{(f)}
$$

и потому в силу сказанного вьше mult $_{o} Y_{(f)} \leqslant \mu \operatorname{deg} f$. Таким образом, можно положить

$$
Z=\mathscr{P}_{c}\left(z_{1}, \ldots, z_{a}\right) \text {. }
$$

Любой ненулевой многочлен $f \in Z$ не содержит целиком ни одну из неприводимых компонент цикла $Y$, так что $Z \backslash\{0\} \subset U_{Y}$. Поэтому $Z \cap \bar{U}_{c}=\{0\}$, как и требовалось.

Рассмотрим теперь общий случай произвольного $k \geqslant 2$. Здесь имеем $k$ точек $o_{1}, \ldots, o_{k} \in \operatorname{Supp} Y$. Рассмотрим систему аффинных функций

$$
\begin{gathered}
l_{1,1}, \ldots, l_{1, a_{1}}, \\
\ldots \ldots \ldots . . \\
l_{k, 1}, \ldots, l_{k, a_{k}},
\end{gathered}
$$

$a_{1}=\cdots=a_{k-1}=a, a_{k}=\min (a, N-(k-1) a)$, удовлетворяюшую следуюшим требованиям:

- линейные части аффинных функций $l_{*, *}$ линейно независимы в совокупности, т.е. образуют часть базиса пространства $\mathbb{C}^{N}$;

- для любого $i \in\{1, \ldots, k-1\}$ имеем $l_{i, \alpha}\left(o_{i}\right)=0$ и система функций $\left(l_{i, 1}, \ldots, l_{i, a}\right)$ правильная для пары $\left(o_{i} \in Y\right) ; l_{k, \alpha}\left(o_{k}\right)=0$ и система функций $\left(l_{k, 1}, \ldots, l_{k, a_{k}}\right)$ образует часть правильного набора для пары $\left(o_{k} \in Y\right)$; - при $i \neq j$ имеем $l_{i, 1}\left(o_{j}\right) \neq 0$.

Положим теперь

$$
Z=\sum_{i=1}^{k}\left(\prod_{j=1}^{i-1} l_{j, 1}^{c_{j}+1}\right) \mathscr{P}_{c_{i}^{*}}\left(l_{i, 1}, \ldots, l_{i, a_{i}}\right),
$$

где $\mathscr{P}_{\alpha}(\#)$ обозначает линейное пространство всех полиномиальных функций степени $\alpha$ от аффинных функций \#. Отметим сразу, что сумма в (35) является прямой, так как линейные части функций $l_{\#}$ линейно независимы. 
ЛЕмма 3.6. Замкнутые алгебраические множества $Z$ и $\bar{U}_{c_{*}}$ пересекаются только по нулю.

ДокаЗАТЕльство. Для $f \in Z$ имеем разложение

$$
f=f_{1}+\cdots+f_{k}, \quad f_{i} \in Z_{i}=\left(\prod_{j=1}^{i-1} l_{j, 1}^{c_{j}+1}\right) \mathscr{P}_{c_{i}^{*}}\left(l_{i, 1}, \ldots, l_{i, a_{i}}\right),
$$

которое определено однозначно, так как $Z=\bigoplus_{i=1}^{k} Z_{i}$.

По построению для любого $i \in\{1, \ldots, k\}$ имеем

$$
\operatorname{mult}_{o_{j}}\left\{\left.f_{i}\right|_{Y}=0\right\} \geqslant\left(c_{j}+1\right) \mu_{j} \geqslant c_{j} \mu_{j}+1
$$

для всех $j \leqslant i-1$. В частности, если $f \in Z \cap \bar{U}_{c_{*}}$, то имеет место оценка

$$
\operatorname{mult}_{o_{1}}\left\{\left.f_{1}\right|_{Y}=0\right\} \geqslant c_{1} \mu_{1}+1 \text {, }
$$

так как для $f_{2}, \ldots, f_{k}$ оценка (36) с $j=1$ справедлива. Рассуждая, как выше, заключаем, что $f_{1} \equiv 0$. Предположим, что уже доказано, что

$$
f_{1} \equiv \cdots \equiv f_{\gamma} \equiv 0
$$

Поскольку при $j=\gamma+1$ оценка (36) выполнена для всех $i \geqslant \gamma+2$, заключаем, что

$$
\operatorname{mult}_{o_{\gamma+1}}\left\{\left.f_{\gamma+1}\right|_{Y}=0\right\} \geqslant c_{\gamma+1} \mu_{\gamma+1}+1 .
$$

Однако по построению

$$
f_{\gamma+1}=\left(\prod_{j=1}^{\gamma} l_{j, 1}^{c_{j}+1}\right) f_{\gamma+1}^{\#}
$$

где $f_{\gamma+1}^{\#} \in \mathscr{P}_{c_{\gamma+1}^{*}}\left(l_{\gamma+1,1}, \ldots, l_{\gamma+1, a_{\gamma+1}}\right)$. Учитывая, что для $j=1, \ldots, \gamma$ имеем $l_{j, 1}\left(o_{\gamma+1}\right) \neq 0$, получаем, что оценка (37) остается справедливой при замене $f_{\gamma+1}$ на $f_{\gamma+1}^{\#}$. Теперь, рассуждая как в случае $k=1$, разобранном выше, заключаем, что $f_{\gamma+1} \equiv 0$. Этим лемма 3.6 доказана.

Учитьвая, что выполнено равенство (34) (это очевидно из явной конструкции (35) линейного пространства $Z$ ), завершаем доказательство леммы 3.5 .

\section{Список литературы}

1. Пухликов $A$. В. Бирационалњно жесткие многообразия с пучком двойных накрытий $\Phi а-$ но. I // Матем. сб. 2004. Т. 195. № 7. С. 127-160.

2. Пухликов $A$. В. Бирациональные автоморфизмы трехмерных алгебраических многообразий с пучком поверхностей дель Пеццо // Изв. РАН. Сер. матем. 1998. Т. 62. № 1. C. $123-164$.

3. Пухликов А.В. Бирационально жесткие расслоения Фано // Изв. РАН. Сер. матем. 2000. T. 64. № 3. C. 131-150.

4. Пухликов A. В. Бирационально жесткие двойные гиперповерхности Фано // Матем. сб. 2000. T. 191. №6. C. 101-126.

5. Pukhlikov A. V. Birational automorphisms of Fano hypersurfaces // Invent. Math. 1998. V. 134. № 2. P. 401-426. 
6. Pukhlikov A. V. Certain examples of birationally rigid varieties with a pencil of double quadrics // J. Math. Sci. 1999. V. 94. № 1. P. 986-995.

7. Пухликов $A$. В. Бирациональные автоморфизмы алгебраических многообразий спучком двойных квадрик // Матем. заметки. 2000. Т. 67. № 2. С. 241-249.

8. Pukhlikov A. V. Essentials of the method of maximal singularities // Explicit birational geometry of threefolds. Cambridge: Cambridge Univ. Press, 2000. P. 73-100. (London Math. Soc. Lecture Note Ser. V. 281.)

9. Pukhlikov A. V. Birationally rigid Fano complete intersections // J. Reine Angew. Math. 2001. V. 541. P. 55-79.

10. Пухликов A. В. Бирационально жесткие итерированные двойные накрытия $\Phi$ ано // Изв. РАН. Сер. матем. 2003. Т. 67. №3. С. 139-182.

11. Гриненко M. М. Бирациональные автоморфизмы трехмерного двойного конуса // Матем. сб. 1998. Т. 189. № 7. С. 37-52.

12. Гриненко $M$. М. Бирациональные свойства пучков поверхностей дель Пеццо степени 1 и 2 // Матем. сб. 2000. Т. 191. № 5. С. 17-38.

13. Гриненко М. М. Бирациональные свойства пучков поверхностей дель Пеццо степени 1 и 2. II // Матем. сб. 2003. Т. 194. № 5. С. 31-60.

14. Соболев И. В. Об одной серии бирационально жестких многообразий с пучком гиперповерхностей Фано // Матем. сб. 2001. Т. 192. № 10. С. 123-130.

15. Соболев И. В. Бирациональные автоморфизмы одного класса многообразий, расслоенных на кубические поверхности // Изв. РАН. Сер. матем. 2002. Т. 66. №1. С. 203-224.

16. Brown G., Corti A., Zucconi F. Birational geometry of 3-fold Morifibre spaces // Preprint 2003; arXiv:math. AG/0307301.

17. Саркисов В.Г. Бирациональные автоморфизмы расслоений на коники // Изв. АН СССР. Сер. матем. 1980. Т. 44. №4. С. 918-945.

18. Саркисов В.Г. О структурах расслоений на коники // Изв. АН СССР. Сер. матем. 1982. T. 46. № 2. C. 371-408.

19. Исковских В.А., Манин Ю.И. Трехмерные квартики и контрпримеры к проблеме Люрота // Матем. сб. 1971. Т. 86. № 1. С. 140-166.

20. Фултон У. Теория пересечений. М.: Мир, 1989.

Математический институт

им. В.А. Стеклова РАН

E-mail: pukh@mi.ras.ru
Поступила в редакцию 12.01 .2004 